\title{
On the combinatorics of the Hopf algebra of dissection diagrams
}

\author{
Cécile Mammez ${ }^{1,2}$
}

Received: 30 October 2018 / Accepted: 21 March 2019 / Published online: 25 May 2019

(c) Springer Science+Business Media, LLC, part of Springer Nature 2019

\begin{abstract}
In this article, we study the Hopf algebra $\mathcal{H}_{\mathrm{D}}$ of dissection diagrams introduced by Dupont in his thesis, more precisely we focus on its underlying coalgebra. We use the version with a parameter $x$ in the base field. We conjecture it is cofree if $x=1$ or $x$ is not a root of unity. If $x=-1$, then we know there is no cofreeness. Since $\mathcal{H}_{\mathrm{D}}$ is a free-commutative right-sided combinatorial Hopf algebra as defined by Loday and Ronco, then there exists a pre-Lie structure on the primitives of its graded dual. Furthermore $\mathcal{H}_{\mathrm{D}}^{\circledast}$ and the enveloping algebra of its primitive elements are isomorphic. Thus, we can equip $\mathcal{H}_{\mathrm{D}}^{\circledast}$ with a structure of Oudom-Guin. We focus on the pre-Lie structure on dissection diagrams and in particular on the pre-Lie algebra generated by the dissection diagram of degree 1 . We prove that it is not free. We express a Hopf algebra morphism between the Grossman-Larson Hopf algebra and $\mathcal{H}_{\mathrm{D}}^{\circledast}$ by using pre-Lie and Oudom-Guin structures.
\end{abstract}

\section{Résumé}

Dans cet article, nous nous intéressons à l'algèbre de Hopf à paramètre $\mathcal{H}_{\mathrm{D}}$ des diagrammes de dissection introduite par Dupont dans sa thèse de doctorat. Nous cherchons plus particulièrement à étudier sa cogèbre sous-jacente. Nous conjecturons qu'elle est colibre si $x=1$ ou $x$ n'est pas une racine de l'unité. Il n'y a pas de coliberté lorsque le paramètre vaut -1 . L'algèbre de Hopf $\mathcal{H}_{\mathrm{D}}$ est un exemple d'algèbre de Hopf combinatoire commutative-associative-libre droite selon Loday et Ronco. Ceci implique l'existence d'une structure pré-Lie sur les primitifs de son dual gradué dont on sait qu'il est isomorphe à l'algèbre enveloppante de ses primitifs. Il est alors possible de munir $\mathcal{H}_{\mathrm{D}}^{\circledast}$ d'une structure de Oudom-Guin. Nous nous intéressons à la structure préLie des diagrammes de dissection et plus particulièrement à la sous-algèbre pré-Lie non triviale engendrée par le diagramme de dissection de degré 1 . Nous montrons que cette dernière n'est pas libre. Nous explicitons un morphisme d'algèbres de Hopf entre celle de Grossman-Larson et $\mathcal{H}_{\mathrm{D}}^{\circledast}$ grâce aux structures pré-Lie et aux structures de Oudom-Guin. 
Keywords Combinatorial Hopf algebras · Dissection diagrams · Cofreeness $\cdot$ Rooted trees $\cdot$ Pre-Lie algebras · Enveloping algebras · Morphism · Insertion process

Mots-clés Algèbres de Hopf combinatoires · diagrammes de dissection · arbres enracinés · algèbres pré-Lie · algèbres enveloppantes · morphisme · procédé d'insertion

Mathematics Subject Classification 16T30 - 05C76

\section{Contents}

Introduction . . . . . . . . . . . . . . . . . . . . . . . . . 480

1 Hopf algebra of dissection diagrams . . . . . . . . . . . . . . . . . . . . . . . . . . . . . 482

1.1 Reminders . . . . . . . . . . . . . . . . . . . . . . . . 482

1.2 Path trees and corollas: two Hopf subalgebras of $\mathcal{H}_{\mathrm{D}} \ldots \ldots \ldots \ldots$. . . . . . . . . . 484

1.2.1 Path trees and symmetric functions . . . . . . . . . . . . . . . . . . . . . . . . . . . . . . . . . . . . . . . . .

1.2.2 Corollas and the Faà di Bruno Hopf algebra . . . . . . . . . . . . . . . . . . . . . . 485

1.3 Primitive elements of degree less than or equal to $3 \ldots \ldots$. . . . . . . . . . . . . . . 486

1.4 The antipode calculation . . . . . . . . . . . . . . . . . . . . . . . 490

2 Dissection diagrams, rooted trees, theorem of Oudom-Guin . . . . . . . . . . . . . . . . 492

2.1 Pre-Lie algebras and theorem of Oudom-Guin . . . . . . . . . . . . . . . . . . . . . . 492

2.2 Hopf algebras of rooted trees . . . . . . . . . . . . . . . . . . . . . . . . 494

2.2.1 Hopf algebra $\mathcal{H}_{\mathrm{GL}}$ of Grossman-Larson rooted trees . . . . . . . . . . . . . . . . . . . . . . . . . . . . . . . . . . .

2.2.2 Quotient Hopf algebra of sub-binary trees . . . . . . . . . . . . . . . . . . . . . . . . . . . . . . . . . . . .

2.3 Dual of the dissection diagrams Hopf algebra . . . . . . . . . . . . . . . . . . . 496

3 Definition of a Hopf algebra morphism from $\mathcal{H}_{\mathrm{GL}}$ to $\mathcal{H}_{\mathrm{D}}^{\circledast} \ldots \ldots$. . . . . . . . . . . . . . 498

3.1 Insertion of a chord in a vertex of a dissection diagram $D \ldots \ldots \ldots 503$

3.1 .1 The chosen vertex of $D$ is different from the root $\ldots \ldots \ldots$. . . . . . . . 503

3.1.2 The chosen vertex of $D$ is its root . . . . . . . . . . . . . . 505

3.1.3 Computation of $L(D)$ where $D$ is a dissection diagram of degree $n \geq 1 \ldots 507$

3.2 Insertion of a chord in two dissection diagrams . . . . . . . . . . . . . . . 511

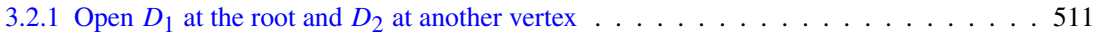

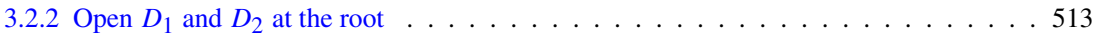

3.2.3 Computation of $L\left(D_{1} D_{2}\right)$ where $\left(D_{1}, D_{2}\right) \in(\mathcal{D})_{n_{1}} \times(\mathcal{D})_{n_{2}}$ and $n_{1}, n_{2} \geq 1 \ldots 516$

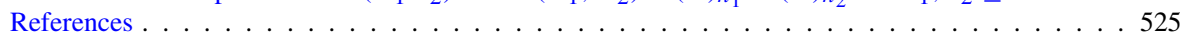

\section{Introduction}

The Hopf algebra of dissection diagrams comes from number theory and was introduced by Dupont [8] [9, Chapter 2]. He considers the coproduct calculation problem in the fundamental Hopf algebra of the category of mixed Hodge-Tate structures. Thanks to dissection diagrams, he aims at computing this coproduct for motivic dissection polylogarithms.

A dissection diagram of degree $n$ is a $(n+1)$-gon with a set of $n$ non-intersecting chords forming a planar rooted tree. Dupont builds a coalgebra structure on the symmetric algebra of dissection diagrams which makes it into a Hopf algebra denoted by $\mathcal{H}_{\mathrm{D}}$. The coproduct is given with a parameter $x$ selection-quotient process [9, Sections 2.1.2 and 2.1.3]. Dupont considers the case $x=-1$, defines a decorated version and explains compatibilities between decorations and chords contraction in an oriented 
graph [9, Section 2.1.4]. The set of decorations must be a group, and in this case, Dupont uses the complex numbers group. For any dissection diagram $D$, he defines dissection polylogarithms $I(D)$ as an absolutely convergent integral of a differential form on a simplex [9, Definition 2.3.5]. The differential form depends on decorated chords and the simplex on decorated sides of $D$ [9, Section 2.3.2]. He gives then a motivic version $I^{\mathcal{H}}(D)[9$, Section 2.4.1] and computes the coproduct [9, Section 2.4.2, Theorem 2.4.9].

In this article, we aim at understanding the combinatorics of the Hopf algebra of dissection diagrams. We first recall the Hopf algebra construction as well as two particular families of dissection diagrams that generate Hopf subalgebras: the path trees and the corollas. We express their Hopf algebraic structures as group coordinate algebras. The path trees subalgebra and the Hopf algebra of symmetric functions are isomorphic. The corollas subalgebra is the dissection diagrams version of the Faà di Bruno Hopf algebra. We then discuss cofreeness. In the case of degree three, we give a basis of the vector space of primitive elements although their dimensions do not permit to reach a conclusion. Computations attest that the Hopf algebra $\mathcal{H}_{\mathrm{D}}$ is not cofree with the parameter $x=-1$. We conclude this first section with calculations of the antipode.

The second part consists in studying $\mathcal{H}_{\mathrm{D}}$ by using Hopf algebras of rooted trees. Sending a dissection diagram to its underlying rooted tree is not a good choice, because it does not respect the coalgebra structure. Dupont [9, Remark 2.1.15] alludes to a preLie structure on dissection diagrams. The Hopf algebra $\mathcal{H}_{\mathrm{D}}^{\circledast}$ is graded, connected, cocommutative, so is isomorphic to the enveloping algebra $\mathcal{U}\left(\mathfrak{g}_{\mathcal{D}}\right)$ of its primitive elements $\mathfrak{g}_{\mathcal{D}}$. According to Loday and Ronco, $\mathcal{H}_{\mathrm{D}}$ is a free-commutative right-sided combinatorial Hopf algebra. This implies that there exists a pre-Lie structure on $\mathfrak{g}_{\mathcal{D}}$. In that way, we can use the Oudom-Guin structural theorem [23, Proposition 2.7 Theorem 2.12] about enveloping algebras of pre-Lie algebras. The goal is then to study the parameterized pre-Lie algebra of dissection diagrams. Indeed, let $x$ be a parameter in the base field. If the pre-Lie algebra of dissection diagrams associated with $x$ is free, then its enveloping algebra is free too and, by duality, the dissection Hopf algebra of dissection diagrams $\mathcal{H}_{\mathrm{D}}$ associated with $x$ is cofree. After a brief recall about pre-Lie algebras and the Grossman-Larson Hopf algebra, we present the Hopf algebra $\mathcal{H}_{\mathrm{D}}^{\circledast}$ and the pre-Lie structure on $\mathfrak{g}_{\mathcal{D}}$. We describe the unique morphism of Hopf algebras $\varphi$ respecting the Oudom-Guin structures of $\mathcal{H}_{\mathrm{GL}}$ and $\mathcal{H}_{\mathrm{D}}^{\circledast}$ and sending the rooted tree $t=$. of degree 1 to $D=($. This morphism relies on an insertion process of chords (Propositions 37, 39, 44 and 46). We prove that the pre-Lie algebra generated by 1 is not free (Corollary 34 ) and is a strict pre-Lie subalgebra of $\mathfrak{g}_{\mathcal{D}}$ (Proposition 29). Consequently, the pre-Lie algebra of dissection diagrams is not free and it does not answer the question of cofreeness. We conjecture the kernel of $\varphi$ to be the Hopf biideal generated by rooted trees with at least one vertex of valency at least three (Conjecture 35). Propositions 41, 48 and 49 are first steps toward a solution to this conjecture. 
This article is a shortened version of chapter 3 of my thesis [21, Chapter 3].

\section{Hopf algebra of dissection diagrams}

\subsection{Reminders}

We recall some notations and the construction of the Hopf algebra of dissection diagrams. Let $\mathbb{K}$ be a commutative base field of characteristic 0 . For any nonnegative integer $n$, we consider a regular oriented $(n+1)$-gon $\Pi_{n}$ with a special vertex called the root. We draw $\Pi_{n}$ as a circle and put the root at the bottom. An arc between two vertices is a side, while a line between two distinct vertices is a chord.

Definition 1 A dissection diagram of degree $n$ is a set of $n$ non-intersecting chords of $\Pi_{n}$ such that the graph formed by the chords is acyclic. So the $n$ chords form a planar rooted tree whose root is the root of $\Pi_{n}$. For any dissection diagram $D$, we denote by $\mathscr{C}(D)$ the set of its chords.

Example We consider the dissection diagram

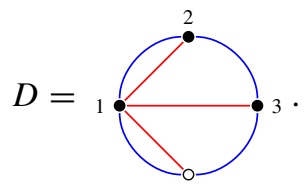

Its sides are colored in blue, and its chords are colored in red.

Lemma 2 The number of dissection diagrams of degree $n \in \mathbb{N}$ is given by

$$
d_{n}=\frac{1}{2 n+1}\left(\begin{array}{c}
3 n \\
n
\end{array}\right)
$$

and satisfies the following recursive relation

$$
\forall n \geq 1, d_{n}=\sum_{\substack{i_{1}, i_{2}, i_{3} \geq 0 \\ i_{1}+i_{2}+i_{3}=n-1}} d_{i_{1}} d_{i_{2}} d_{i_{3}} .
$$

Proof Let us recall ideas expounded by Dupont [9, Lemma 2.1.1] in his proof. For any dissection diagram $D$, there exist a unique triple of dissection diagrams $\left(D_{1}, D_{2}, D_{3}\right)$ and a unique triple of integers $\left(i_{1}, i_{2}, i_{3}\right)$ such that $i_{1}+i_{2}+i_{3}=n-1$ and for any $j \in\{1,2,3\}$ the dissection diagram $D_{j}$ is of degree $i_{j}$ and $D$ is the dissection diagram

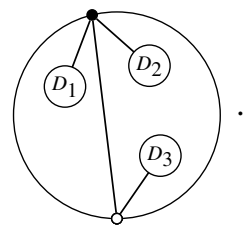


The black vertex is the first vertex, in the clockwise orientation, connected with the root. So, if $d(h)=\sum_{n \geq 0} d_{n} h^{n}$, then

$$
d(h)=1+h d(h)^{3}
$$

By the Lagrange inversion formula, for any integer $n \in \mathbb{N}, d_{n}$ is then given by

$$
d_{n}=\frac{1}{n}\left\langle(1+h)^{3 n}, h^{n-1}\right\rangle=\frac{1}{2 n+1}\left(\begin{array}{c}
3 n \\
n
\end{array}\right),
$$

where $\left\langle(1+h)^{3 n}, h^{n-1}\right\rangle$ is the coefficient of $h^{n-1}$ in $(1+h)^{3 n}$.

The decomposition of dissection diagrams presented in the proof of Lemma 2 will be useful to prove Propositions 41 and 48

Now we denote by $\mathcal{D}$ the vector space spanned by dissection diagrams. The formal series $d$ ([25, Sequence A001764 ]) recalled in the preceding proof is the PoincaréHilbert series of $\mathcal{D}$.

Since a dissection diagram $D$ is clockwise oriented, it is possible to label the sides of $\Pi_{n}$. Furthermore, since the chords of $D$ form a planar rooted tree, it is possible to orient them toward the root and to label them. If it is necessary, we label the root by 0 .

\section{Example}

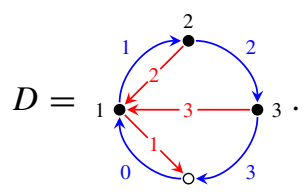

It is thus possible to do the following identification

$$
\mathscr{C}(D) \simeq\{1, \ldots, n\} \simeq \mathscr{S}^{+} \text {where } \mathscr{S}^{+}=\left\{\text {sides of } \Pi_{n}\right\} \backslash\{\text { side } 0\}
$$

Since this identification is natural, quite often we do not write any label to lighten the notation.

Let $D$ be a dissection diagram and $C$ be a subset of $\mathscr{C}(D)$. We assume that the cardinality of $C$ is $p$. Chords in $C$ give a partition of $\Pi_{\operatorname{deg}(D)}$ in $p+1$ faces. For any face $\alpha, \mathscr{S}_{C}(\alpha)$ is the set of sides of $\Pi_{\operatorname{deg}(D)}$ which are in the face $\alpha$. We shall consider the set $\mathscr{S}_{C}^{+}(D)=\sqcup_{\alpha} \mathscr{S}_{C}^{+}(\alpha)$ where $\mathscr{S}_{C}^{+}(\alpha)=\mathscr{S}_{C}(\alpha) \backslash\left\{\min \left(\mathscr{S}_{C}(\alpha)\right)\right\}$.

Definition 3 Consider $\mathcal{H}_{\mathrm{D}}$, which is, as vector space, the symmetric algebra generated by $\mathcal{D}$ (i.e. as vector space $\mathcal{H}_{\mathrm{D}}=\mathcal{S}(\mathcal{D})$ ), and define the two maps $m$ and $\Delta$ by:

$$
m:\left\{\begin{aligned}
\mathcal{H}_{\mathrm{D}} \otimes \mathcal{H}_{\mathrm{D}} & \longrightarrow \mathcal{H}_{\mathrm{D}} \\
D_{1} \otimes D_{2} & \longrightarrow D_{1} D_{2}
\end{aligned}\right.
$$


and

$$
\Delta:\left\{\begin{aligned}
\mathcal{H}_{\mathrm{D}} & \longrightarrow \mathcal{H}_{\mathrm{D}} \otimes \mathcal{H}_{\mathrm{D}} \\
D & \longrightarrow \sum_{C \subset \mathscr{C}(D)} x^{k_{C}(D)} q_{C}(D) \otimes r_{C}(D)
\end{aligned}\right.
$$

where

1. $x \in \mathbb{K}$ is scalar,

2. $q_{C}(D)$ is the disjoint union obtained by contracting chords in $C$,

3. $r_{C}(D)$ is the dissection diagram obtained by keeping chords in $C$ and contracting sides of $\Pi_{\operatorname{deg}(D)}$ which are in the set $\mathscr{S}_{C}^{+}(D)$,

4. $k_{C}(D)$ is the number of changes of direction we need to properly orient chords of $r_{C}(D)$.

Dupont proves in [9, Proposition 2.1.11] the following proposition.

Proposition 4 1. The map $\Delta$ is a coassociative coproduct.

2. The quintuple $\left(\mathcal{H}_{\mathrm{D}}, m, 1_{\mathcal{H}_{\mathrm{D}}}, \Delta, \varepsilon\right)$, where $1_{\mathcal{H}_{\mathrm{D}}}$ is the unit of $\mathcal{H}_{\mathrm{D}}$ and $\varepsilon$ its counit, is a Hopf algebra.

Example For example, we have the following coproduct:

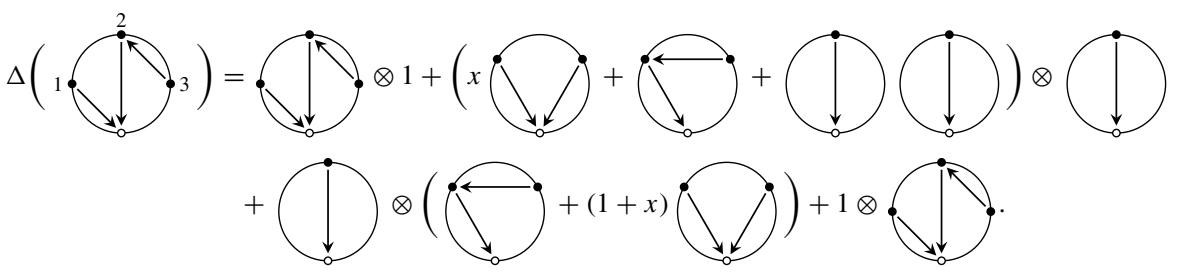

\subsection{Path trees and corollas: two Hopf subalgebras of $\mathcal{H}_{\mathrm{D}}$}

Dupont [9, Examples 2.1.14] emphasizes two special families: the path trees and the corollas. In fact, they form two Hopf subalgebras which are isomorphic to group coordinate Hopf algebras. We recall these families and explain the isomorphisms.

\subsubsection{Path trees and symmetric functions}

Let $n$ be a positive integer. The path tree of degree $n$ is the dissection diagram $Y_{n}$ of degree $n$ such that for any $i \in \llbracket 1, n-1 \rrbracket$, chord $i$ goes from vertex $i$ to vertex $i+1$ and chord $n$ connects vertex $n$ to the root. The path tree of degree 0 is the empty dissection diagram i.e. $Y_{0}=1$.

Examples Path trees of degree 1-4:
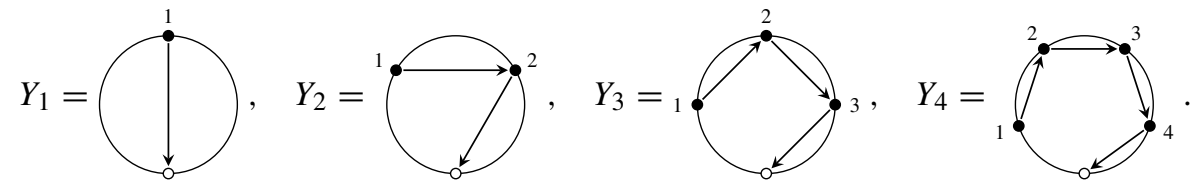
For any nonnegative integer $n$, we have

$$
\Delta\left(Y_{n}\right)=\sum_{k=0}^{n}\left(\begin{array}{l}
n \\
k
\end{array}\right) Y_{k} \otimes Y_{n-k}
$$

Trivially, the vector space generated by disjoint unions of path trees is a Hopf subalgebra of $\mathcal{H}_{\mathrm{D}}$ here denoted by $\mathcal{E}_{Y}$. Dupont [9, Examples $\left.2.1 .14,1\right]$ remarks that the $\mathcal{E}_{Y}$ coproduct is reminiscent of the one on symmetric functions. We give an isomorphism between both.

Let $G_{0}=\left\langle 1+\sum_{n=1}^{\infty} q_{n} h^{n} \in \mathbb{K}[[h]]\right\rangle$ be the multiplicative group of formal series with constant term equal to 1 . For any positive integer $n$, we call $n$-th coordinate map the map $\Sigma_{n}$ defined by $\Sigma_{n}:\left\{\begin{array}{l}\mathbb{K}[[h]] \longrightarrow \mathbb{K} \\ Q=1+\sum_{n=1}^{\infty} q_{n} h^{n} \longrightarrow q_{n} .\end{array}\right.$ We consider the Hopf algebra $S y m=\mathbb{K}\left[\Sigma_{1}, \ldots, \Sigma_{n}, \ldots\right]$ with its usual product and the coproduct given by: for any map $f \in S y m$, for any elements $P$ and $Q$ of $G_{0}, \Delta_{G_{0}}(f)(P \otimes Q)=f(P Q)$.

Proposition 5 The Hopf algebras $\mathcal{E}_{Y}$ and Sym are isomorphic.

Proof It is sufficient to consider the following Hopf algebra morphism

$$
\omega_{1}:\left\{\begin{aligned}
S y m & \longrightarrow \mathcal{E}_{Y} \\
\Sigma_{n} & \longrightarrow \frac{1}{n !} Y_{n} .
\end{aligned}\right.
$$

Remark Actually, the morphism defined in the proof above comes from the one used by DOUBILET, RoTA and STANLEY to prove that the reduced incidence algebra of the poset of path trees and the algebra of exponential power series [7, Example 4.6].

\subsubsection{Corollas and the Faà di Bruno Hopf algebra}

The second special family emphasized by Dupont is that of the corollas. For any positive integer $n$, the corolla of degree $n$ is the dissection diagram such that for any $i \in \llbracket 1, n \rrbracket$ the chord $i$ goes from vertex $i$ to the root. The corolla of degree 0 is the empty dissection diagram i.e. $X_{0}=1$.

Examples Non-empty corollas of degree $n$ less than or equal to 4:
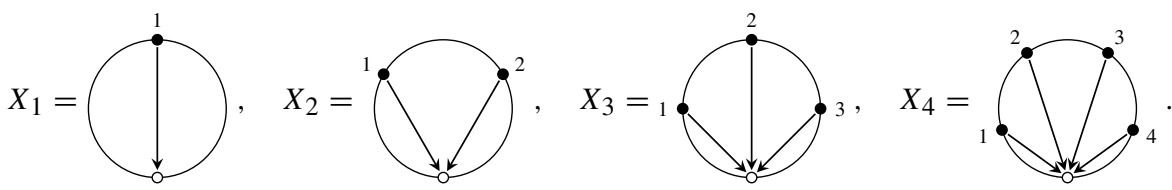
For any nonnegative integer $n$, we have:

$$
\Delta\left(Y_{n}\right)=\sum_{k=0}^{n}\left(\begin{array}{l}
n \\
k
\end{array}\right) Y_{k} \otimes Y_{n-k}
$$

Trivially, the vector space spanned by disjoint union of corollas is a Hopf subalgebra of $\mathcal{H}_{\mathrm{D}}$ that we denote by $\mathcal{C}_{X}$.

Let us now recall the Faà di Bruno Hopf algebra construction. Let us consider the set $G_{1}=\left\langle h+\sum_{n=1}^{\infty} q_{n} h^{n+1} \in \mathbb{K}[[h]]\right\rangle$. Endowed with the natural formal series composition $\circ$, it is the group of formal diffeomorphisms tangent to identity. For any positive integer $n$, we still call $n$-th coordinate map the linear map $\Sigma_{n}$ defined by

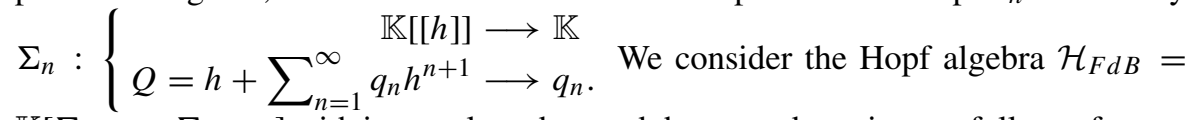
$\mathbb{K}\left[\Sigma_{1}, \ldots, \Sigma_{n}, \ldots\right]$ with its usual product and the coproduct given as follows: for any map $f \in \mathcal{H}_{F d B}$ and elements $P$ and $Q$ of $G_{1}, \Delta_{G_{1}}(f)(P \otimes Q)=f(Q \circ P)$. It is the Faà di Bruno Hopf algebra.

Proposition 6 The Hopf algebras $\mathcal{C}_{X}$ and $\mathcal{H}_{F d B}$ are isomorphic.

Proof It is sufficient to consider the morphism

$$
\omega_{2}:\left\{\begin{aligned}
\mathcal{H}_{F d B} & \longrightarrow \mathcal{C}_{X} \\
\Sigma_{n} & \longrightarrow X_{n}
\end{aligned}\right.
$$

\subsection{Primitive elements of degree less than or equal to 3}

We want to study the underlying coalgebra of $\mathcal{H}_{\mathrm{D}}$ to determine whether it is cofree or not. We start by giving for any parameter $x \in \mathbb{K}$ a basis of the vector space $\operatorname{Prim}\left(\mathcal{H}_{\mathrm{D}}\right)_{2}$ (respectively $\left.\operatorname{Prim}\left(\mathcal{H}_{\mathrm{D}}\right)_{3}\right)$ spanned by degree 2 (respectively degree 3 ) primitive elements. Unfortunately, these two cases are not sufficient to decide whether $\mathcal{H}_{\mathrm{D}}$ is cofree or not.

Let $F_{\mathcal{H}_{\mathrm{D}}}$ be the Poincaré-Hilbert formal series of $\mathcal{H}_{\mathrm{D}}$. By definition, $F_{\mathcal{H}_{\mathrm{D}}}(h)=$ $\prod_{n=1}^{\infty} \frac{1}{\left(1-h^{n}\right)^{d_{n}}}$, where $d_{n}$ is the number of dissection diagrams of degree $n$ for any nonnegative integer. If $\mathcal{H}_{\mathrm{D}}$ is cofree, we have $F_{\text {Prim }\left(\mathcal{H}_{\mathrm{D}}\right)}=1-\frac{1}{F_{\mathcal{H}_{\mathrm{D}}}}$ i.e.

$$
F_{\operatorname{Prim}\left(\mathcal{H}_{\mathrm{D}}\right)}(h)=h+3 h^{2}+9 h^{3}+40 h^{4}+185 h^{5}+\cdots
$$

A basis of $\operatorname{Prim}\left(\mathcal{H}_{\mathrm{D}}\right)_{2}$ is given by the following triple $\left(V_{1}, V_{2}, V_{3}\right)$ of independent vectors where

$$
V _ { 1 } = ( 1 + x ) \Longrightarrow - 2 \longdiv { \square },
$$




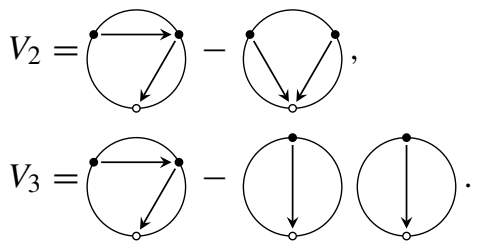

Definition 7 We define the map $\kappa$ by

$$
\kappa:\left\{\begin{aligned}
\mathcal{H}_{\mathrm{D}} \otimes \mathcal{H}_{\mathrm{D}} & \longrightarrow \mathcal{H}_{\mathrm{D}} \otimes \mathcal{H}_{\mathrm{D}} \\
U_{1} \otimes U_{2} & \longrightarrow U_{2} \otimes U_{1}
\end{aligned}\right.
$$

The morphism $\kappa \circ \Delta$ is denoted by $\Delta^{o p}$.

For the third degree case, we add the following notations.

Definition 8 Let $U$ be in $\mathcal{H}_{\mathrm{D}}$.

1. We denote by $l(U)$ its projection over $\mathcal{D}^{+}$, by $q(U)$ its projection over $\left(\mathcal{D}^{+}\right)^{2}$, by $t(U)$ its projection over $\left(\mathcal{D}^{+}\right)^{3}$ and by $r(U)$ the sum $U-l(U)-q(U)-t(U)$.

2. We call linear part of $\Delta(U)$ the projection of $\Delta(U)$ over $\mathcal{D}^{+} \otimes \mathcal{D}^{+}$denoted by $\delta(U)$.

3. The opposite linear part of $\Delta(U)$ is the linear part of $\Delta^{o p}(U)$ denoted by $\delta^{o p}(U)$.

4. We call quadratic part of $\Delta(U)$ the projection of $\Delta(U)$ over $\left(\mathcal{D}^{+}\right)^{2} \otimes \mathcal{D}^{+}$denoted by $\mathcal{Q}(U)$.

5. The opposite quadratic part of $\Delta(U)$ is the quadratic part of $\Delta^{o p}(U)$ denoted by $\mathcal{Q}^{o p}(U)$.

Lemma 9 Let $p$ be a primitive element of degree 3. It can be written as $p=l(p)+$ $q(p)+t(p)$ and we have:

$$
\begin{aligned}
m \circ \delta(l(p)) & =-2 q(p), \\
\mathcal{Q}(l(p)) & =0, \\
m \circ \mathcal{Q}(t(p)) & =-3 t(p) .
\end{aligned}
$$

Proof Let $p$ be a primitive element of degree 3. By commutativity, we have $m \circ$ $\delta(l(p))=-2 q(p)$. By definition of $t(p)$ and since $p$ is of degree 3 , there exists a scalar $k \in \mathbb{K}$ such that $t(p)=k\left(C_{0}^{1} C_{0}^{1}\right.$. Similarly, there exist scalars $k_{1}, k_{2}$ and $k_{3}$ in $\mathbb{K}$ such that $q(p)=k_{1}$ 
$k_{3} \overbrace{0}^{1} \overbrace{0}^{1}$. So $\mathcal{Q}(t(p))=\mathcal{Q}^{o p}(t(p))$ and $\mathcal{Q}(q(p))=\mathcal{Q}^{o p}(q(p))$. This finally gives $\mathcal{Q}(l(p))=0$.

Lemma 10 Let $p$ be a primitive element of degree 3. The part $l(p)$ is a linear combination of the independent vectors $U_{1}, \ldots, U_{9}$ where
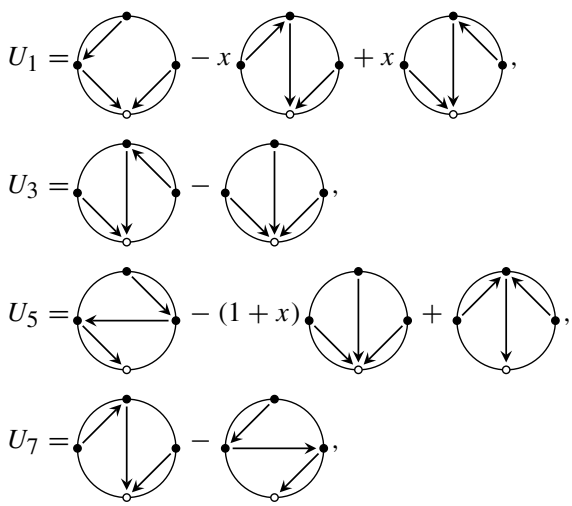
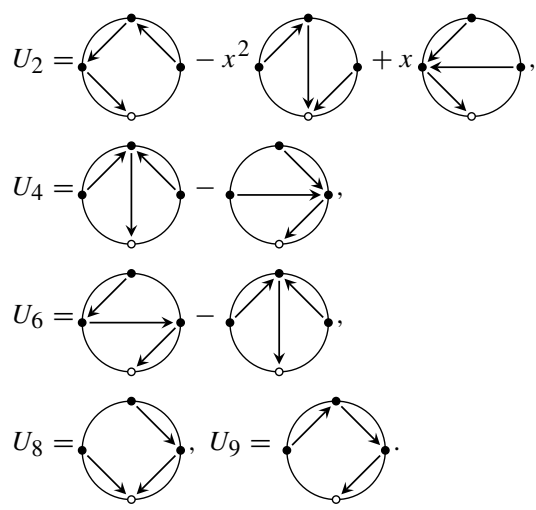

Proposition 11 A basis of $\operatorname{Prim}\left(\mathcal{H}_{\mathrm{D}}\right)_{3}$ is given by the vector family $\left\{V_{1}, \ldots, V_{9}\right\}$ where

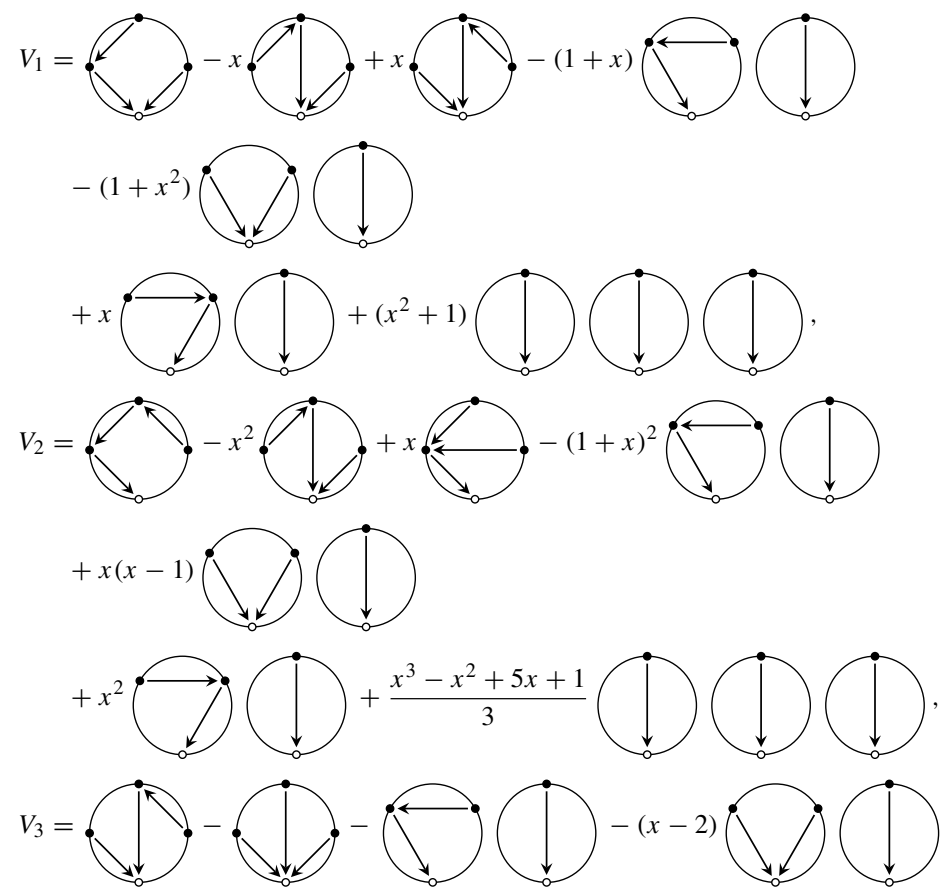



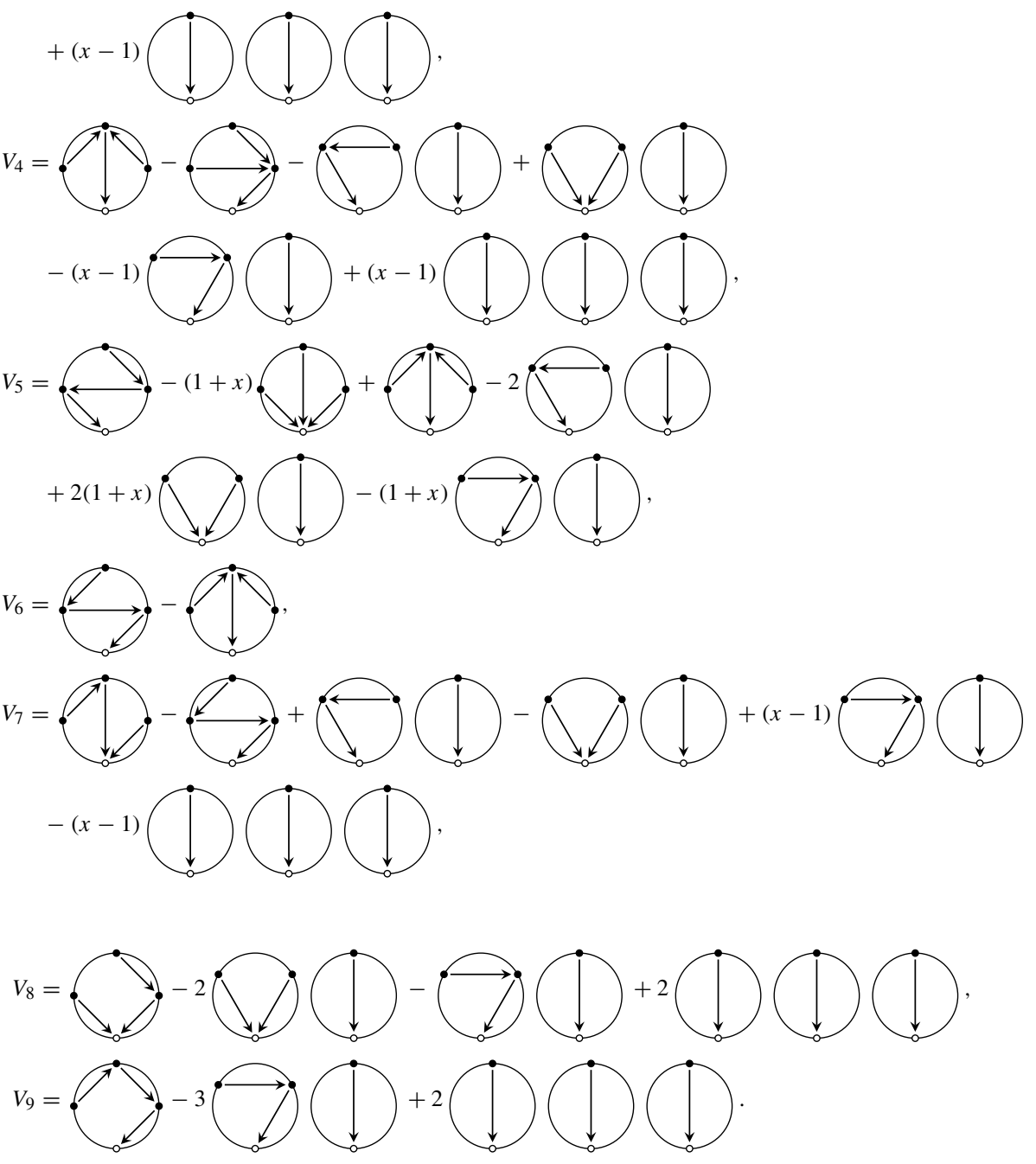

The bases given before are compatible with formula (1). We use the computation program in [21, Appendix B.3 ] to determine the dimension of primitives elements of low degree for some parameterized Hopf algebras $\mathcal{H}_{\mathrm{D}}$. With those computations, it is possible to explain that $\mathcal{H}_{\mathrm{D}}$ is not cofree if $x=-1$. Indeed, in degree 5 , if $x=-1$, the primitive space dimension equals 187 which is different from 185 the dimension compatible with cofreeness. If $x \in \llbracket-10^{4}, 10^{4} \rrbracket$, the dimensions of the primitive spaces of degree $d \in \llbracket 1,4 \rrbracket$ are compatible with cofreeness. In degree 5 , because of a long computation time, we only calculated the dimension of the vector space for $x \in \llbracket-100,100 \rrbracket$. Except for $x=-1$, all dimensions turn to be equal to 185.

Conjecture 12 If $x=1$ or $x$ is not a root of unity, the Hopf algebra $\mathcal{H}_{\mathrm{D}}$ is cofree. 


\subsection{The antipode calculation}

We want to give a formula for the antipode. We first have to understand iterated coproducts i.e. morphisms $\Delta^{k}=(\Delta \otimes \underbrace{I d \otimes \cdots \otimes I d}_{k-1 \text { times }}) \circ \cdots \circ(\Delta \otimes I d) \circ \Delta$ where $k$ is a positive integer. Let $D$ be a dissection diagram and let $C$ be a subset of $\mathscr{C}(D)$. By contracting $C$, we change labels in $q_{C}(D)$ and $r_{C}(D)$. As we build iterated coproducts by applying the coproduct on the left, we need to modify the labels of the chords of $q_{C}(D)$. It is sufficient to label the chords of $q_{C}(D)$ with the labels they had in $D$ before the contraction. In the sequel, we keep the same notation for $q_{C}(D)$ and for the element $q_{C}(D)$ with modified labels.

Proposition 13 Let $n$ be a positive integer and $D$ be a dissection diagram of degree $n$. The value of the antipode $S$ evaluated at $D$ is given by:

$$
S(D)=\sum_{s=1}^{n}(-1)^{s} \sum_{P \in \Pi_{D}(s)} x^{k_{P}(D)} \prod_{i=1}^{s} r_{C_{i}}\left(q_{P_{i-1}}(D)\right)
$$

where

$\Pi_{D}(s)$ is the set of $s$-tuples $\left(C_{1}, \ldots, C_{s}\right)$ of non-empty sets which are a partition of $\mathscr{C}(D)$,

$P_{0}=\emptyset$ for any $P$ in $\Pi_{D}(s)$,

$P_{i}=\bigcup_{u=1}^{i} C_{u}$ for any $P$ in $\Pi_{D}(s)$ and each integer $i$ in $\llbracket 1, s \rrbracket$,

$k_{P}(D)=k_{C_{1}}\left(q_{P_{0}}(D)\right)+\cdots+k_{C_{s}}\left(q_{P_{s-1}}(D)\right)$.

If, for any nonnegative integer $p$, we denote by $X_{p}$ (respectively by $Y_{p}$ ) the corolla (respectively the path tree) of degree $n$, we have in particular: 


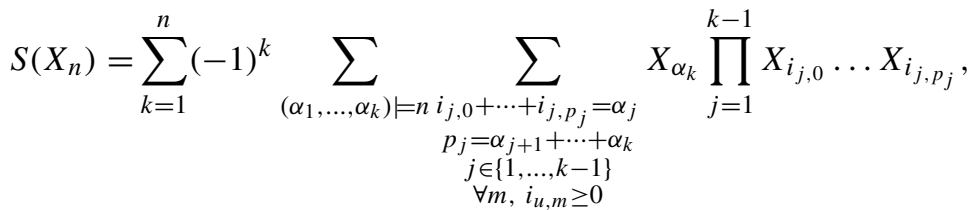

$$
\begin{aligned}
& S\left(Y_{n}\right)=\sum_{k=1}^{n}(-1)^{k} \sum_{\alpha=\left(\alpha_{1}, \ldots, \alpha_{k}\right) \models n} \frac{n !}{\alpha_{1} ! \ldots \alpha_{k} !} Y_{\alpha_{1}} \ldots Y_{\alpha_{k}}
\end{aligned}
$$

Proof Let $n$ be a positive integer and $D$ be a dissection diagram of degree $n$. For any integer $s$ in $\llbracket 1, n-1 \rrbracket$, we define

$$
\begin{aligned}
\tilde{\Pi}_{D}(s)= & \left\{\left(C_{1}, \ldots, C_{s+1}\right) \in \mathscr{C}(D)^{s+1},\left(C_{1}, \ldots, C_{s}\right) \in \Pi_{D}(s)\right. \text { or } \\
& \left.\left(C_{1}, \ldots, C_{s+1}\right) \in \Pi_{D}(s+1)\right\} .
\end{aligned}
$$

We use the fact that the antipode reverses the identity map for the convolution product and on each step we use the renumbering process described earlier. Using a reasoning by induction, this gives:

$$
\begin{aligned}
S(D) & =-\sum_{\substack{C_{1} \subset \mathscr{C}(D) \\
C_{1} \neq \emptyset}} x^{k_{C_{1}}(D)} S\left(q_{C_{1}}(D)\right) r_{C_{1}(D)} \\
& =\sum_{\substack{C_{1} \subset \mathscr{C}(D) \\
C_{1} \neq \emptyset}} x^{k_{C_{1}}(D)} \sum_{\substack{C_{2} \subset \mathscr{C}(D) \backslash C_{1} \\
C_{2} \neq \emptyset}} x^{k_{C_{2}}\left(q_{C_{1}}(D)\right)} S\left(q_{C_{1} \cup C_{2}}(D)\right) r_{C_{2}}\left(q_{C_{1}}(D)\right) r_{C_{1}(D)} \\
& =(-1)^{u} \sum_{P \in \tilde{\Pi}_{D}(u)} x^{k_{P}(D)} S\left(q P_{u}(D)\right) \prod_{i=1}^{u} r_{C_{i}}\left(q P_{i-1}(D)\right) \\
& =\sum_{s=1}^{n}(-1)^{s} \sum_{P \in \Pi_{D}(s)} x^{k_{P}(D)} \prod_{i=1}^{s} r_{C_{i}}\left(q_{P_{i-1}}(D)\right) .
\end{aligned}
$$

\section{Examples}
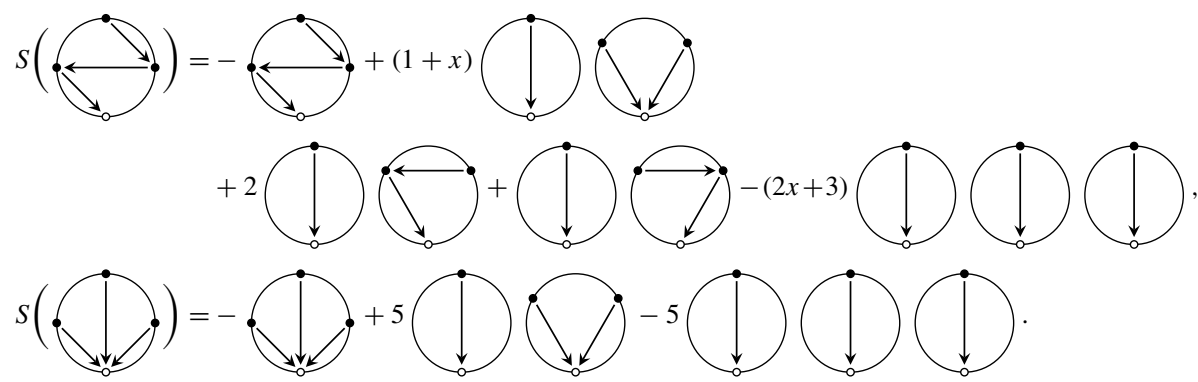
Remark Considering the Faà di Bruno Hopf algebra as the Hopf algebra of coordinates of the group of formal diffeomorphisms $G_{1}$, for any positive integer $n$, the antipode $S$ computes in the coordinate map $\Sigma_{n}$ is given by:

$$
\forall P \in G_{1}, S\left(\Sigma_{n}\right)(P)=\Sigma_{n}\left(P^{-1}\right)
$$

where $P^{-1}$ is the inverse of $P$ for the formal series composition [13, Section 4.3].

\section{Dissection diagrams, rooted trees, theorem of Oudom-Guin}

The aim of this section is to connect dissection diagrams and rooted trees. For instance, we grade rooted trees by the number of edges and associate with a disjoint union of dissection diagrams the disjoint union of underlying planar rooted trees. Unfortunately, this procedure does not respect the coalgebra structure.

Dupont alludes to a pre-Lie structure over the primitive elements $\mathfrak{g}_{\mathcal{D}}$ of $\mathcal{H}_{\mathrm{D}}^{\circledast}[9$, Remark 2.1.15]. We therefore consider $\mathcal{H}_{\mathrm{D}}^{\circledast}$ the enveloping algebra of the pre-Lie algebra $\mathfrak{g}_{\mathcal{D}}$ and apply the structural theorem of Oudom-Guin [23, Proposition 2.7 Theorem 2.12]. This allows us to build the unique pre-Lie morphism $\gamma$ from the free pre-Lie algebra $\mathfrak{g}_{\mathcal{T}_{\mathrm{R}}}$ generated by $t=$. to $\mathfrak{g}_{\mathcal{D}}$ sending $t$ to the dissection diagram of degree 1 . From this, we deduce that the pre-Lie algebra generated by the dissection diagram of degree 1 is not free and does not generate the whole pre-Lie algebra $\mathfrak{g}_{\mathcal{D}}$. We extend $\gamma$ to a morphism of Hopf algebras and give a conjecture about its kernel.

\subsection{Pre-Lie algebras and theorem of Oudom-Guin}

Pre-Lie algebras (also called Vinberg algebras) were introduced in 1963 by Vinberg [27] in the theory of homogeneous convex cones and by Gerstenhaber [14, Section $2]$ in deformation theory. The $\operatorname{Pr} e \mathcal{L} i e$ operad defining pre-Lie algebras was introduced and described by Chapoton and Livernet [5, Theorem 1.9]. They also describe the free pre-Lie algebra generated by one or several generators in terms of rooted trees [5, Corollaire 1.10]. It is another way to prove the isomorphism between the dual of the Connes and Kreimer Hopf algebra [6] and the Grossman-Larson Hopf algebra [15]. Foissy proves that the free pre-Lie algebra with one generator is free as a Lie algebra [11, Theorem 8.4]. Using operad theory, Chapoton extended this result for any free pre-Lie algebra [4, Corollary 5.3]. He proves that the operad Pre $\mathcal{L}$ ie is anticyclic [3]. Livernet [19, Theorem 3.4] determines the freedom of "Hopf pre-Lie algebras" equipped the relation $\Delta(x \triangleright y)=\Delta(x) \triangleright y+x \otimes y=$ $x^{(1)} \triangleright y \otimes x^{(2)}+x^{(1)} \otimes x^{(2)} \triangleright y+x \otimes y$. Oudom and Guin [23, Proposition 2.7 - Theorem 2.12] build for any pre-Lie algebra $\mathfrak{g}$ a Hopf algebra which is isomorphic to $\mathcal{U}(\mathfrak{g})$. It is a generalization of the construction of the enveloping algebra of the free pre-Lie algebra of rooted trees generated by $t=$. (Grossman-Larson Hopf algebra). Loday and Ronco [20, Theorems 5.3 and 5.8] explain that there exists a pre-Lie structure for free-commutative right-sided combinatorial Hopf algebras. It is possible to equip an operad with a pre-Lie structure. Burgunder, Delcroix-Oger 
and Manchon [2, Theorem 3.1] attest that an operad cannot be free as a pre-Lie algebra.

New structures can be defined too. Mansuy builds the quadratic operad $\mathcal{C}$ om $\mathcal{P}$ re $\mathcal{L}$ ie and gives as an example the algebra of rooted trees equipped with the grafting product and the grafting product on the root [22, Section 4.2]. Foissy [12, Definition 17 and Theorem 20] describes the free $\mathcal{C}$ om - Pre $\mathcal{L}$ ie algebra with one generator as the partitioned trees algebra equipped with the disjoint union product and with the grafting product. Another structure of interest is the quadratic operad Post $\mathcal{L}$ ie introduced by Vallette [26, Section A.2]. A post-Lie algebra $\mathcal{A}$ is equipped with a binary map $\triangleright$ and with a Lie bracket $\{-,-\}$ which are compatible. If $(\mathcal{A},\{-,-\})$ is abelian, then $(\mathcal{A}, \triangleright)$ is a pre-Lie algebra. The post-Lie algebra notion is a generalization of the pre-Lie algebra notion. A general survey about the origins and applications of pre-Lie algebras can be found in [1].

Definition 14 A left pre-Lie algebra is a couple $(\mathfrak{g}, \triangleright)$ where $\mathfrak{g}$ is a vector space and $\triangleright: \mathfrak{g} \otimes \mathfrak{g} \longrightarrow \mathfrak{g}$ is an internal product with the following relation: for any $x, y, z \in \mathfrak{g}$,

$$
x \triangleright(y \triangleright z)-(x \triangleright y) \triangleright z=y \triangleright(x \triangleright z)-(y \triangleright x) \triangleright z .
$$

Example We consider the classical example of $\mathfrak{g}=\{P(X) \partial, P(X) \in \mathbb{K}[X]\}$, the derivation algebra of $\mathbb{K}[X]$, where $\partial$ is the derivation sending $X$ to 1 . We define the product $\triangleright$ by

$$
\triangleright:\left\{\begin{aligned}
\mathfrak{g} \otimes \mathfrak{g} & \longrightarrow \mathfrak{g} \\
P(X) \partial \otimes Q(X) \partial & \longrightarrow(P(X) \partial Q(X)) \partial .
\end{aligned}\right.
$$

The product of two derivations $P(X) \partial$ and $Q(X) \partial$ is not the usual composition of maps ( $\mathfrak{g}$ is not stable under this product) but is the unique derivation sending $X$ to $P(X) Q^{\prime}(X)$. Let $P(X), Q(X)$ and $R(X)$ be polynomials. We have

$$
P(X) \partial \triangleright(Q(X) \partial \triangleright R(X) \partial)-(P(X) \partial \triangleright Q(X) \partial) \triangleright R(X) \partial=P(X) Q(X) \partial^{2} R(X) \partial .
$$

This relation is symmetric over $P(X)$ and $Q(X)$, so the pre-Lie relation is satisfied.

By symmetrization, a pre-Lie algebra is a Lie algebra.

Proposition 15 Let $(\mathfrak{g}, \triangleright)$ be a pre-Lie algebra. We define the bracket $\{-,-\}$ by:

$$
\{-,-\}:\left\{\begin{array}{l}
\mathfrak{g} \otimes \mathfrak{g} \longrightarrow \mathfrak{g} \\
x \otimes y \longrightarrow x \triangleright y-y \triangleright x .
\end{array}\right.
$$

With this bracket, $\mathfrak{g}$ is a Lie algebra denoted by $\mathfrak{g}_{\text {Lie }}$.

Definition 16 Let $(\mathfrak{g}, \triangleright)$ be a pre-Lie algebra. Consider the Hopf symmetric algebra $\mathcal{S}(\mathfrak{g})$ equipped with its usual coproduct $\Delta$. The product $\triangleright$ can be extended to $\mathcal{S}(\mathfrak{g})$. Let $a, b, c$ and $x$ be elements such that $a, b, c \in \mathcal{S}(\mathfrak{g})$ and $x \in \mathfrak{g}$. One defines 


$$
\left\{\begin{aligned}
1 \triangleright a & =a, \\
a \triangleright 1 & =\varepsilon(a) 1, \\
(x a) \triangleright b & =x \triangleright(a \triangleright b)-(x \triangleright a) \triangleright b, \\
a \triangleright(b c) & =\sum_{a}\left(a^{(1)} \triangleright b\right)\left(a^{(2)} \triangleright c\right) .
\end{aligned}\right.
$$

On $\mathcal{S}(\mathfrak{g})$, we define a product $\star$ by:

$$
\star\left\{\begin{aligned}
\mathcal{S}(\mathfrak{g}) \otimes \mathcal{S}(\mathfrak{g}) & \longrightarrow \mathcal{S}(\mathfrak{g}) \\
a \otimes b & \longrightarrow a \star b=\sum_{a} a^{(1)}\left(a^{(2)} \triangleright b\right) .
\end{aligned}\right.
$$

The following theorem was proved by Oudom and Guin [23, Proposition 2.7 Theorem 2.12].

Theorem 17 The space $(\mathcal{S}(\mathfrak{g}), \star, \Delta)$ is a Hopf algebra which is isomorphic to the enveloping Hopf algebra $\mathcal{U}\left(\mathfrak{g}_{\text {Lie }}\right)$ of the Lie algebra $\mathfrak{g}_{\text {Lie }}$ generated by primitive elements.

\subsection{Hopf algebras of rooted trees}

\subsubsection{Hopf algebra $\mathcal{H}_{\mathrm{GL}}$ of Grossman-Larson rooted trees}

The Grossman-Larson Hopf algebra, written $\mathcal{H}_{\mathrm{GL}}$, was introduced in [15] as a tool in the theory of differential operators $[16,17]$. It is graded, connected, cocommutative and not commutative. By the Cartier-Quillen-Milnor-Moore theorem, it is isomorphic to the enveloping algebra of its primitive elements. Panaite [24] proves that there exists a connection between $\mathcal{H}_{\mathrm{GL}}$ and the graded dual $\mathcal{H}_{\mathrm{CK}}^{\circledast}$ of the Connes and Kreimer Hopf algebra [6]. Hoffman clarifies this connection [18]. The two Hopf algebras are not equal but isomorphic in characteristic 0 . In $\mathcal{H}_{\mathrm{CK}}^{\circledast}$, because of grafts, there are symmetry coefficients which do not appear in $\mathcal{H}_{\mathrm{GL}}$. Chapoton and Livernet [5, Corollary 1.10] prove that the pre-Lie algebra of rooted trees in Grossman-Larson Hopf algebra is free and give another proof of the isomorphism between $\mathcal{H}_{\mathrm{GL}}$ and $\mathcal{H}_{\mathrm{CK}}^{\circledast}$. Oudom and Guin [23, Proposition 2.7 - Theorem 2.12] use the case of $\mathcal{H}_{\mathrm{GL}}$ as model to prove a structural theorem for the enveloping algebra of a pre-Lie algebra.

We recall the definition of $\mathcal{H}_{\mathrm{GL}}$ with the point of view of Oudom and Guin. Let $\mathfrak{g}_{\mathcal{T}_{\mathrm{R}}}$ be the vector space $\mathfrak{g}_{\mathcal{T}_{\mathrm{R}}}=\operatorname{Vect}\left(t, t \in \mathcal{T}_{\mathrm{R}}\right)$.

Definition 18 On $\mathfrak{g}_{\mathcal{T}_{\mathrm{R}}}$ we define the following product:

$$
\triangleright:\left\{\begin{aligned}
\mathfrak{g}_{\mathcal{T}_{\mathrm{R}}} \otimes \mathfrak{g}_{\mathcal{T}_{\mathrm{R}}} & \longrightarrow \mathfrak{g}_{\mathcal{T}_{\mathrm{R}}} \\
t_{1} \otimes t_{2} & \longrightarrow \sum_{s \in V\left(t_{2}\right)} \tau_{t_{1}, t_{2}, s}
\end{aligned}\right.
$$

where $\tau_{t_{1}, t_{2}, s}$ is the rooted tree obtained by grafting $t_{1}$ on the vertex $s$ of $t_{2}$.

The following theorem was proved by Chapoton and Livernet [5, Corollary 1.10]. 
Theorem 19 The algebra $\left(\mathfrak{g}_{\mathcal{T}_{\mathrm{R}}}, \triangleright\right)$ is the free left pre-Lie algebra generated by the rooted tree of degree 1 (i.e. $t=$.).

\section{Examples}

$$
: \triangleright \dot{l}=\ddot{v}+\ddot{\gtrless}+\vdots, \quad \vdots \triangleright:=\dot{v}+\vdots
$$

Proposition 20 On $\mathcal{S}\left(\mathfrak{g}_{\mathcal{T}_{\mathrm{R}}}\right)$, the product $\star$, built with the pre-Lie structure and the theorem of Oudom-Guin, is given by:

$\star:\left\{\begin{aligned} \mathcal{H}_{\mathrm{GL}} \otimes \mathcal{H}_{\mathrm{GL}} & \longrightarrow \mathcal{H}_{\mathrm{GL}} \\ t_{1} \ldots t_{n} \otimes t_{n+1} \ldots t_{n+m} & \longrightarrow \sum_{\sigma: I \subseteq \llbracket 1, n \rrbracket \longrightarrow V\left(t_{n+1} \ldots t_{n+m}\right)}\left(t_{1} \ldots t_{n}, t_{n+1} \ldots t_{n+m}, \sigma\right)\end{aligned}\right.$

where

$V\left(t_{n+1} \ldots t_{n+m}\right)$ is the set of the vertices of the forest $t_{n+1} \ldots t_{n+m}$,

$\left(t_{1} \ldots t_{n}, t_{n+1} \ldots t_{n+m}, \sigma\right)$ is the rooted forest obtained by grafting the tree $t_{i}$ on the vertex $\sigma(i)$ for any $i$ in $I$.

\section{Examples}

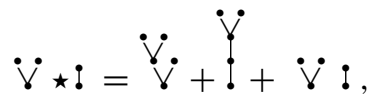

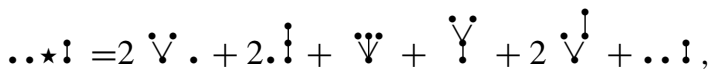

$$
\begin{aligned}
& \text { } \star \ldots=2 . !+\ldots \text {. }
\end{aligned}
$$

\subsubsection{Quotient Hopf algebra of sub-binary trees}

Definition 21 1. A rooted tree $t$ is a sub-binary tree if all its vertices have a valency less than or equal to 3 . The set of rooted sub-binary trees is denoted by $\mathcal{T}_{\mathrm{SB}}$.

2. A rooted forest $F$ is sub-binary if all of its trees are sub-binary trees. We denote the set of rooted sub-binary forests by $\mathcal{F}_{\mathrm{SB}}$.

By direct checking, we obtain the following proposition.

Proposition 22 We consider the vector space $\mathcal{I}=\operatorname{Vect}\left(F, F \in \mathcal{F}_{\mathrm{R}} \backslash \mathcal{F}_{\mathrm{SB}}\right)$. It is a Hopf biideal of $\mathcal{H}_{\mathrm{GL}}$.

Proposition 23 The vector space $\mathbf{S B T}=\operatorname{Vect}\left(F, F \in \mathcal{F}_{\mathrm{SB}}\right)$ is a quotient Hopfalgebra of $\mathcal{H}_{\mathrm{GL}}$. 
Proof It is sufficient to consider the canonical surjection

$$
\Pi_{\text {SBT }}:\left\{\begin{array}{c}
\mathcal{H}_{\mathrm{GL}} \longrightarrow \text { SBT } \\
F \in \mathcal{F}_{\mathrm{R}} \longrightarrow \begin{cases}F & \text { if } F \in \mathcal{F}_{\mathrm{SB}}, \\
0 & \text { else. }\end{cases}
\end{array}\right.
$$

The biideal $\mathcal{I}$ is the kernel of $\Pi_{\mathbf{S B T}}$.

Remark By duality, we can consider SBT ${ }^{\circledast}$ as a Hopf subalgebra of the Connes and Kreimer Hopf algebra.

\subsection{Dual of the dissection diagrams Hopf algebra}

As the Hopf algebra $\mathcal{H}_{\mathrm{D}}$ is graded and connected with finite-dimensional homogeneous components, its graded dual $\mathcal{H}_{\mathrm{D}}^{\circledast}$ is a graded connected with finite-dimensional homogeneous components Hopf algebra. We now study this structure. Up until Corollary 26, we use the same approach as in [10, Chapter 7, Section 7.3].

Thanks to the Cartier-Quillen-Milnor-Moore theorem, we have the following proposition.

Proposition 24 The Hopf algebra $\mathcal{H}_{\mathrm{D}}^{\circledast}$ is isomorphic to the enveloping algebra $\mathcal{U}\left(\mathfrak{g}_{\mathcal{D}}\right)$ where $\mathfrak{g}_{\mathcal{D}}$ is the Lie algebra $\operatorname{Prim}\left(\mathcal{H}_{\mathrm{D}}^{\circledast}\right)$ of the primitive elements of $\mathcal{H}_{\mathrm{D}}^{\circledast}$.

We denote by $\left(Z_{F_{D}}\right)_{F_{D} \in \mathcal{H}_{\mathrm{D}}}$ the dual basis of disjoint union of dissection diagrams. We know that generators of the Lie algebra $\operatorname{Prim}\left(\mathcal{H}_{\mathrm{D}}^{\circledast}\right)$ are $\mathfrak{g}_{\mathcal{D}}=\left((1)+\operatorname{Ker}(\varepsilon)^{2}\right)^{\perp}$, so a basis of $\mathfrak{g}_{\mathcal{D}}$ is given by $\left(Z_{D}\right)_{D \in \mathcal{D}}$. In order to describe operations of the Hopf algebra $\mathcal{H}_{\mathrm{D}}^{\circledast}$, it is sufficient to define the product on $Z_{D}$, with $D$ a dissection diagram. Each dissection diagram $D$ is primitive. Concerning the product, we have the following proposition:

Proposition 25 Let $D_{1}$ and $D_{2}$ be two non-empty dissection diagrams of respective degree $n_{1}$ and $n_{2}$ and $x$ a scalar. The product of $Z_{D_{1}}$ and $Z_{D_{2}}$ in this order is given by:

$$
Z_{D_{1}} Z_{D_{2}}=\left(1+\delta_{D_{1}, D_{2}}\right) Z_{D_{1} D_{2}}+\sum_{D \in \mathcal{D}} c\left(D_{1}, D_{2} ; D\right) Z_{D}
$$

where for any dissection diagram $D$, the coefficient $c\left(D_{1}, D_{2} ; D\right)$ is a polynomial $Q_{D}$ in $x$ depending on $D$. We have:

$$
Q_{D}(x)=\left(Z_{D_{1}} \otimes Z_{D_{2}}\right) \circ \Delta(D) .
$$

\section{Example}

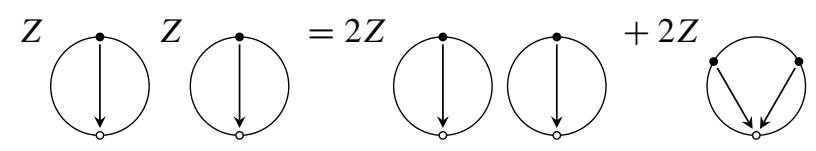




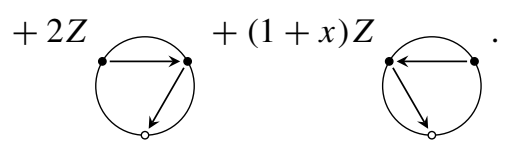

Corollary 26 The Lie algebra $\mathfrak{g}_{\mathcal{D}}$ is the vector space $\mathfrak{g}_{\mathcal{D}}=\operatorname{Prim}\left(\mathcal{H}_{\mathrm{D}}^{\circledast}\right)$ equipped with the bracket $[-,-]$ defined, for any dissection diagrams $D_{1}$ and $D_{2}$, by:

$$
\left[Z_{D_{1}}, Z_{D_{2}}\right]=\sum_{D \in \mathcal{D}}\left(c\left(D_{1}, D_{2} ; D\right)-c\left(D_{2}, D_{1} ; D\right)\right) Z_{D}
$$

\section{Example}

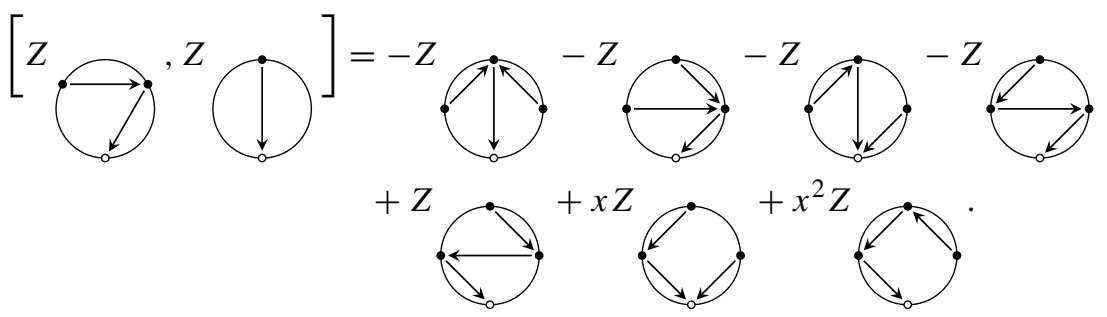

Now we aim at defining a Hopf algebra isomorphic to $\mathcal{H}_{\mathrm{D}}^{\circledast}$ by providing it with a structure of Oudom-Guin. We then create a morphism between $\mathcal{H}_{\mathrm{GL}}$ and $\mathcal{H}_{\mathrm{D}}^{\circledast}$ using pre-Lie structures.

Proposition 27 The vector space $\mathfrak{g}_{\mathcal{D}}=\operatorname{Prim}\left(\mathfrak{g}_{\mathcal{D}}\right)$, equipped with $\triangleright$ defined by:

$$
\triangleright:\left\{\begin{aligned}
\mathfrak{g}_{\mathcal{D}} \otimes \mathfrak{g}_{\mathcal{D}} & \longrightarrow \mathfrak{g}_{\mathcal{D}} \\
Z_{D_{1}} \otimes Z_{D_{2}} & \longrightarrow \sum_{D \in \mathcal{D}} c\left(D_{1}, D_{2} ; D\right) Z_{D},
\end{aligned}\right.
$$

is a left pre-Lie algebra.

Proof Let us first recall the notion of free-commutative right-sided combinatorial Hopf algebra introduced by Loday and Ronco [20, Definitions 3.16, 4.1, 4.16 and 5.7]. A free-commutative right-sided combinatorial Hopf algebra $H$ is an associative commutative free Hopf algebra $(H, m, \Delta)$ generated by $\operatorname{Gen}(H)$ and such that, for $h \in \operatorname{Gen}(H)$, we have $\Delta(h)=\sum_{h} h^{(1)} \otimes h^{(2)}$ with $h^{(2)} \in \operatorname{Gen}(H)$. The Hopf algebra $\mathcal{H}_{\mathrm{D}}$ is a free-commutative right-sided combinatorial Hopf algebra. According to Loday and Ronco [20, Theorems 5.3 and 5.8], the couple $\left(\mathfrak{g}_{\mathcal{D}}, \triangleright\right)$ is a left pre-Lie algebra.

Proposition 28 We consider the Hopf algebra $\mathcal{S}=\left(\mathcal{S}\left(\mathfrak{g}_{\mathcal{D}}\right), \star, \Delta\right)$ where $\mathcal{S}\left(\mathfrak{g}_{\mathcal{D}}\right)$ is the symmetric algebra of dissection diagrams, the coproduct $\Delta$ is the usual coproduct of $\mathcal{S}\left(\mathfrak{g}_{\mathcal{D}}\right)$, and the product $\star$ is induced by the pre-Lie product $\triangleright$ defined on $\mathfrak{g}_{\mathcal{D}}$ and Definition 16. The Hopf algebras $\mathcal{S}$ and $\mathcal{H}_{\mathrm{D}}^{\circledast}$ are isomorphic.

Proof It is a direct application of Theorem 17 and proposition 24. We now identify $\mathcal{H}_{\mathrm{D}}^{\circledast}$ with the Hopf algebra obtained by the theorem of Oudom-Guin. 
Lemma 29 The pre-Lie subalgebra generated by the dissection diagram

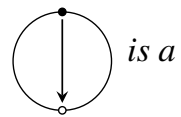
non-trivial pre-Lie subalgebra of $\mathfrak{g}_{\mathcal{D}}$.

Proof The underlying vector subspace of elements of degree 2 of the pre-Lie algebra generated by<smiles>IC1CCCCCC1</smiles>
is generated by

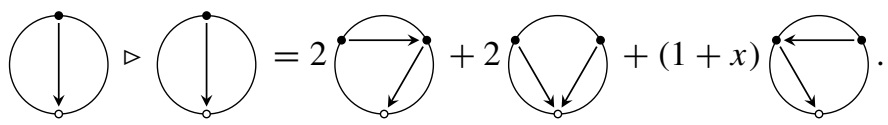

And yet, the underlying vector space of elements of degree 2 of $\mathfrak{g}_{\mathcal{D}}$ is generated by the three dissection diagrams of degree 2 . As a consequence, the pre-Lie subalgebra generated by the dissection diagram (i) is a non-trivial pre-Lie subalgebra of $\mathfrak{g}_{\mathcal{D}}$.

\section{Definition of a Hopf algebra morphism from $\mathcal{H}_{\mathrm{GL}}$ to $\mathcal{H}_{\mathrm{D}}^{\circledast}$}

We want to describe a Hopf algebra morphism between the Grossman-Larson rooted forests Hopf algebra and the graded dual of the Hopf algebra of dissection diagrams. Therefore, we use the underlying pre-Lie structure of the two algebras, the Hopf algebra structure and a chords insertion process. To lighten the notation for any disjoint union $U$, the element $Z_{U}$ of $\mathcal{H}_{\mathrm{D}}^{\circledast}$ is denoted by $U$.

Remark If $f: \mathcal{H}_{\mathrm{GL}} \longrightarrow \mathcal{H}_{\mathrm{D}}^{\circledast}$ is a graded Hopf morphism homogeneous of degree $k \geq 0$, then it is not surjective. Indeed, for any positive integers different from 1 there are less rooted forests of degree $n$ than dissection diagrams of degree $n$. Let $n$ be a positive integer. We denote by $C_{n}$ the $n$-th Catalan number. There exist at most $C_{n}$ rooted forests of degree $n$. We denote by $d_{n}$ the number of dissection diagrams of degree $n$. Then, $C_{1}=d_{1}$ and, if $n$ is greater than 2, then we have:

$$
\frac{d_{n}}{C_{n}}=\frac{(n+1)\left(\begin{array}{c}
3 n \\
n
\end{array}\right)}{(2 n+1)\left(\begin{array}{c}
2 n \\
n
\end{array}\right)}=\frac{(3 n) !(n+1) !}{(2 n+1) !(2 n) !}=\frac{(2 n+2) \ldots(3 n)}{(n+2) \ldots(2 n)}
$$

Definition 30 We call $\gamma$ the following pre-Lie morphism:

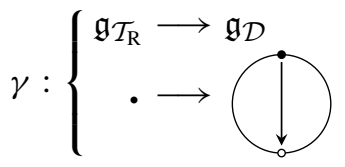


Now we just have to extend this pre-Lie morphism $\gamma$ as a Hopf algebra morphism. Let us first recall the definition of the grafting operator.

Definition 31 The grafting operator, denoted by $B$, is given by:

$$
B:\left\{\begin{aligned}
\mathbb{K}\left[\mathcal{T}_{\mathrm{R}}\right] & \longrightarrow \mathfrak{g}_{\mathcal{T}_{\mathrm{R}}} \\
t_{1}, \ldots, t_{n} \in \mathcal{T}_{\mathrm{R}} & \longrightarrow \text { the rooted tree obtained by grafting } t_{1}, \ldots, t_{n} \text { on a common root. }
\end{aligned}\right.
$$

Example $B\left(t_{1}, t_{2}\right)={ }^{t_{1}} \ddot{\vartheta}^{t_{2}}={ }^{t_{2}} \ddot{\vartheta}^{t_{1}}=B\left(t_{2}, t_{1}\right)$

Proposition 32 The unique extension $\varphi$ of $\gamma$, built with the Oudom-Guin structure of $\mathcal{H}_{\mathrm{GL}}$, is defined by:

$$
\varphi:\left\{\begin{aligned}
\mathcal{H}_{\mathrm{GL}} & \longrightarrow \mathcal{H}_{\mathrm{D}}^{\circledast} \\
t_{1} \ldots t_{n} & \longrightarrow \varphi\left(t_{1}\right) \ldots \varphi\left(t_{n}\right) \\
t & \longrightarrow L\left(\varphi\left(t_{1}\right) \ldots \varphi\left(t_{k}\right)\right)
\end{aligned}\right.
$$

where $t_{1} \ldots t_{k}$ is the rooted forest such that $t=B\left(t_{1} \ldots t_{k}\right)$ and $L$ is the following linear map:

$$
L:\left\{\begin{array}{c}
\mathcal{H}_{\mathrm{D}} \longrightarrow \mathcal{D} \\
D_{1} \ldots D_{n} \longrightarrow D_{1} \ldots D_{n} \triangleright(\square)
\end{array}\right.
$$

Proof Let $t_{1} t_{2}$ be a rooted forest of 2 rooted trees. We have $t_{1} t_{2}=t_{1} * t_{2}-t_{1} \triangleright t_{2}$, so $\varphi\left(t_{1} t_{2}\right)=\varphi\left(t_{1}\right) \varphi\left(t_{2}\right)$. We now assume that there exists a positive integer $n \geq$ 2 such that for any rooted forest $t_{1} \ldots t_{n}$ of $n$ rooted trees we have $\varphi\left(t_{1} \ldots t_{n}\right)=$ $\varphi\left(t_{1}\right) \ldots \varphi\left(t_{n}\right)$. Let $t_{1} \ldots t_{n+1}$ be a rooted forest of $n+1$ rooted trees. We have:

$$
\begin{aligned}
t_{1} \ldots t_{n+1}= & t_{1} * t_{2} \ldots t_{n+1}-\left(t_{1} \triangleright t_{2}\right) t_{3} \ldots t_{n+1}-t_{2} \ldots t_{n}\left(t_{1} \triangleright t_{n+1}\right) \\
& -\sum_{i=2}^{n-1} t_{2} \ldots t_{i}\left(t_{1} \triangleright t_{i+1}\right) t_{i+2} \ldots t_{n+1}
\end{aligned}
$$

Then, by using the fact that $\varphi$ respects the Oudom-Guin structure and the induction hypothesis, $\varphi\left(t_{1} \ldots t_{n+1}\right)=\varphi\left(t_{1}\right) \ldots \varphi\left(t_{n+1}\right)$.

Let $t$ be a rooted tree of degree at least 2. There exist a unique positive integer $k$ and a $k$-tuple $\left(t_{1}, \ldots, t_{k}\right)$ such that $t=B\left(t_{1}, \ldots, t_{k}\right)$. Oudom and Guin observe that the grafting operator $B$ corresponds to the extended pre-Lie product of a rooted forest on the tree of degree 1. It follows that $t=B\left(t_{1}, \ldots, t_{k}\right)=t_{1} \ldots t_{k} \triangleright$. and $\varphi(t)=L\left(\varphi\left(t_{1}\right) \ldots \varphi\left(t_{k}\right)\right)$.

Corollary 33 Let t be a rooted tree. Ift has at least one vertex of valency strictly greater than three, then $\gamma(t)=0$, so $\varphi$ is not an injective morphism. 
Proof It is sufficient to prove the statement:

$$
\forall n \in \mathbb{N}^{*} \backslash\{1,2\}, \forall\left(D_{1}, \ldots, D_{n}\right) \in\left(\mathcal{D}^{+}\right)^{n}, D_{1} \ldots D_{n} \triangleright \square_{\square}=0
$$

We prove the result by induction. Let $U$ and $V$ be two dissection diagrams. We recall that $I d$ is the identity morphism and $l$ is the projection on $\mathcal{D}^{+}$. By definition,

$$
\begin{aligned}
& L(U V)=U V \triangleright(\downarrow)=U \triangleright(V \triangleright(\square))-(U \triangleright V) \triangleright\left(\prod_{0}\right) \\
& =\sum_{\substack{G \in \mathcal{D} \\
G \text { diagram }}}\left(\left(Z_{U} \otimes Z_{V} \otimes Z\left(\square_{0}\right) \circ(I d \otimes \Delta) \circ \Delta\right)(G) G\right. \\
& -\sum_{\substack{G \in \mathcal{D} \\
G \text { diagram }}}\left(\left(Z_{U} \otimes Z_{V} \otimes Z \bigodot^{\circ}\right) \circ(\Delta \otimes I d) \circ(l \otimes I d) \circ \Delta\right)(G) G \\
& =\left\{\begin{array}{l}
\sum_{\substack{G \in \mathcal{D} \\
G \text { diagram }}}\left(Z_{U V} \otimes Z\right)(\Delta(G)) G \quad \text { if } U \neq V, \\
2 \sum_{\substack{G \in \mathcal{D} \\
G \text { diagram }}}\left(Z_{U V} \otimes Z\right.
\end{array}\right.
\end{aligned}
$$

Let $D_{1}, D_{2}$ and $D_{3}$ be three dissection diagrams. We have:

$$
\begin{aligned}
D_{1} D_{2} D_{3} \triangleright\left(\prod_{0}=\right. & D_{1} \triangleright\left(D_{2} D_{3} \triangleright\left(D_{0}\right)-\left(D_{1} \triangleright D_{2}\right) D_{3} \triangleright{ }_{0}\right) \\
& -D_{2}\left(D_{1} \triangleright D_{3}\right) \triangleright\left(\square_{0}\right)
\end{aligned}
$$

By the previous calculation, $D_{1} D_{2} D_{3} \triangleright \bigcirc=0$.

We now assume that there exists a positive integer $n$ greater than or equal to 3 such that for any $n$-tuple $\left(D_{1}, \ldots, D_{n}\right)$ of dissection diagrams $D_{1} \ldots D_{n} \triangleright \square=0$. 
Let $D_{1}, \ldots, D_{n+1}$ be $n+1$ dissection diagrams. We have:

$$
\begin{aligned}
& D_{1} \ldots D_{n+1} \triangleright(\downarrow)=D_{1} \triangleright\left(D_{2} \ldots D_{n+1} \triangleright(\square)\right) \\
& -\left(D_{1} \triangleright D_{2} \ldots D_{n+1}\right) \triangleright\left(\prod_{0}\right) \\
& =D_{1} \triangleright\left(D_{2} \ldots D_{n+1} \triangleright\left(\prod_{0}\right)\right. \\
& -\left(D_{1} \triangleright D_{2}\right) D_{3} \ldots D_{n+1} \triangleright\left(\square_{0}\right. \\
& -\sum_{i=2}^{n-1} D_{2} \ldots D_{i}\left(D_{1} \triangleright D_{i+1}\right) D_{i+2} \ldots D_{n+1} \triangleright \\
& -D_{2} \ldots D_{n}\left(D_{1} \triangleright D_{n+1}\right) \triangleright( \\
& =0
\end{aligned}
$$

Corollary 34 The pre-Lie algebra generated by

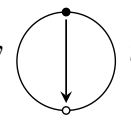
is not free, the pre-Lie algebra

$\mathfrak{g}_{\mathcal{D}}$ is not free either.

Proof The first result is a direct consequence of Corollary 33. For the second one, we assume $\mathfrak{g}_{\mathcal{D}}$ is free as a pre-Lie algebra. Let $V$ be the vector space such that $\mathfrak{g}_{\mathcal{D}}$ is the pre-Lie algebra freely generated by $V$. Let $\mathcal{B}$ be a basis of $V$. By Chapoton and Livernet [5, Corollary 1.10], $\mathfrak{g}_{\mathcal{D}}$ is isomorphic to the pre-Lie algebra of rooted trees decorated by $\mathcal{B}$ with the grafting product. Since there is just one dissection diagram of degree 1 , it is an element of $\mathcal{B}$. As a consequence, the pre-Lie algebra generated by is isomorphic to the pre-Lie algebra of undecorated rooted trees with the grafting product and so it is a free pre-Lie algebra, which is impossible.

Conjecture 35 We call $\mathcal{N}$ the vector space defined by:

$$
\mathcal{N}=\operatorname{Vect}\left(F \in \mathcal{F}_{\mathrm{R}}, \exists s \in V(F), \operatorname{val}(s) \geq 4\right)
$$

i.e. the vector space generated by the non sub-binary forests. Then, $\mathcal{N}$ is the kernel of the Hopf algebra morphism $\varphi$. 
We now consider, as starting space, the quotient Hopf algebra SBT of sub-binary rooted trees. We keep the same notation $\varphi$ for this new morphism. We have:

$$
\varphi:\left\{\begin{aligned}
\mathbf{S B T} & \longrightarrow \mathcal{H}_{\mathrm{D}}^{\circledast} \\
B(t) \in \mathcal{T}_{\mathrm{SB}} & \longrightarrow L(\varphi(t)), \\
B\left(t_{1} t_{2}\right) \in \mathcal{T}_{\mathrm{SB}} & \longrightarrow L\left(\varphi\left(t_{1}\right) \varphi\left(t_{2}\right)\right), \\
t_{1} \ldots t_{n} \in \mathcal{F}_{\mathrm{SB}} & \longrightarrow \varphi\left(t_{1}\right) \ldots \varphi\left(t_{n}\right),
\end{aligned}\right.
$$

where $L$ is the following linear map:

$$
L:\left\{\begin{aligned}
\mathcal{D}^{+} \otimes \mathbb{K} \oplus \mathcal{D}^{+} \otimes \mathcal{D}^{+} & \longrightarrow \mathcal{D}^{+} \\
D \otimes 1 & \longrightarrow D \triangleright \\
D_{1} \otimes D_{2} & \longrightarrow D_{1} D_{2} \triangleright
\end{aligned}\right.
$$

Examples We consider the rooted tree $t=:$. We know that $t=B(\bullet)$, so we have:

$$
\varphi ( t ) = L ( ( ) ) = 2 \longdiv { \square } + 2 \bigcap _ { 0 } + ( 1 + x )
$$

For the rooted tree $t=\ddot{\vee}=B(.$.$) , we have:$

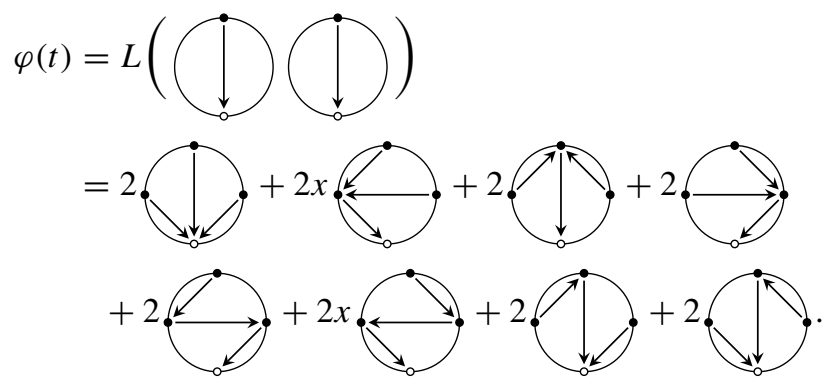

By construction, we have:

Lemma 36 Let $t$ be a sub-binary rooted tree of positive degree n. Its image $\varphi(t)$ is homogeneous of degree $n$.

We now aim at determining $L(D)$ and $L\left(D_{1} D_{2}\right)$ for any dissection diagrams $D, D_{1}$ and $D_{2}$. To this end, we formalize the dissection diagram construction by an insertion process of a chord in one or two dissection diagrams. Let $D$ be a dissection diagram of degree $n \geq 1$ and $i$ an integer in the interval $\llbracket 0, n \rrbracket$.

- The integer $i$ is the vertex $i$ (or the root, if $i=0$ ). If it is necessary for understanding, then the vertex $i$ is denoted by $S_{D, i}$. 
- We call valency of the vertex $i$ the number $v_{D}(i)$ of chords of $D$ attached to the vertex $i$.

- The set of the chords of $D$ containing the vertex $i$ in common, counterclockwise labeled, is defined by: $A_{D, i}=\left\{a_{D, 1}^{i}, \ldots, a_{D, v_{D}(i)}^{i}\right\}$.

- Let $u<v$ be integers between 0 and $n$. We denote by $\{u, v\}$ a chord connecting the vertices $u$ and $v$. As the dissection diagram has a natural orientation, we do not recall the orientation of the chord $\{u, v\}$ in its notation.

- We consider an integer $t \in \llbracket 0, v_{D}(i) \rrbracket$. We define two subsets of chords: $A_{D, i}^{t, 1}=$ $\left\{a_{D, 1}^{i}, \ldots, a_{D, t}^{i}\right\}$ and $A_{D, i}^{t, 2}=\left\{a_{D, t+1}^{i}, \ldots, a_{D, v_{D}(i)}^{i}\right\}$.

If there is no ambiguity on the considered dissection diagram, we do not write its name in notations.

Examples We illustrate the way of labeling chords with a common vertex. In the first example below, we consider a vertex different from the root and color it in red. In the second one, we consider the root and we color it in yellow. In the two cases, chords with the considered common vertex are colored in red. To lighten the notations, the $s^{t h}$ chords from the vertex $i$ is written $s$ instead of $a_{D, s}^{i}$.
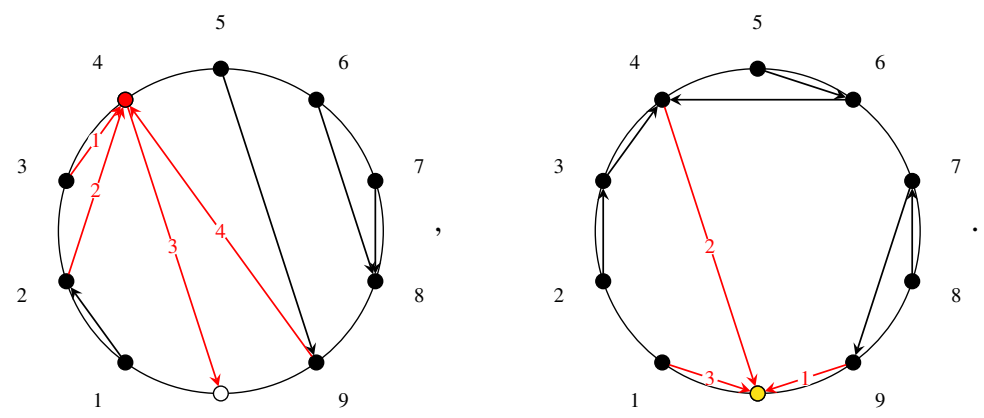

\subsection{Insertion of a chord in a vertex of a dissection diagram $D$}

We consider $D$ a dissection diagram of degree $n \geq 1$. We want to insert a new chord in $D$. Therefore, we start by choosing a vertex $i$ in $D$, we split $i$ in two vertices $s_{1}$ and $s_{2}$, and we split the chords of $D$ connected to $i$. The new object is not a dissection diagram. It is sufficient to build the chord between $s_{1}$ and $s_{2}$ to obtain again a dissection diagram. Following this procedure, we can build all dissection diagrams $G$ of degree $n+1$ with at least one chord $a$ such that $q_{\{a\}}(G)=D$.

\subsubsection{The chosen vertex of $D$ is different from the root}

We choose a vertex of $D$ different from its root, so we just consider a positive integer $i$ in $\llbracket 1, n \rrbracket$. Let $t \in \llbracket 0, f(i) \rrbracket$ be a integer, used to split the chord of $D$ with the common 
vertex $i$. We define the following map:

$$
\phi_{i, t}:\left\{\begin{array}{l}
\mathscr{C}(D) \longrightarrow\{\{u, v\}, 0 \leq u<v \leq n+1\} \\
\{u, v\} \longrightarrow \begin{cases}\{u, v\} & \text { if }(u \leq i-1 \text { and } v \leq i-1) \\
\{u, v+1\} & \text { or }\left(u \leq i-1, v=i \text { and }\{u, v\} \in A_{i}^{t, 1}\right), \\
& \text { if }\left(u \leq i-1, v=i \text { and }\{u, v\} \in A_{i}^{t, 2}\right) \\
\{u+1, v+1\} & \text { or }\left(u=i \text { and }\{u, v\} \in A_{i}^{t, 1}\right),\end{cases}
\end{array}\right.
$$

With this map, we consider a diagram $\tilde{G}_{D, i, t}$ of degree $n+1$ which is open between the vertices $i$ and $i+1$ with $\mathscr{C}\left(\tilde{G}_{D, i, t}\right)=\phi_{i, t}(\mathscr{C}(D))$. This new diagram is not a dissection diagram, but an intermediate object in the definition of the insertion process.

Examples We consider some dissection diagrams $D$ and give their diagram $\tilde{G}_{D, i, t}$. The vertex $i$ to split is colored in red, the chords of $D$ in $A_{D, i}^{t, 1}$ are colored in blue, and the chords in $A_{D, i}^{t, 2}$ are colored in green.
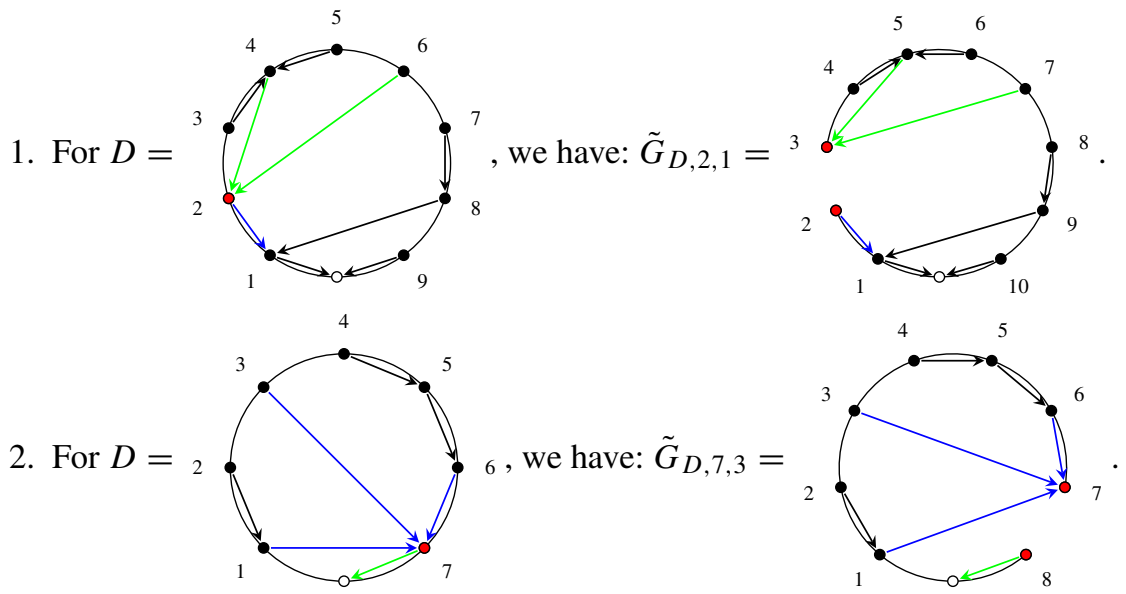

It is then possible to define an insertion endomorphism homogeneous of degree 1 of the vector space of dissection diagrams. We consider a positive integer $i$ (choice of the vertex to make the insertion) and a nonnegative integer $t$ (partition of the chord with the vertex $i$ in common). We define the map $\Gamma_{i, t}$ by:

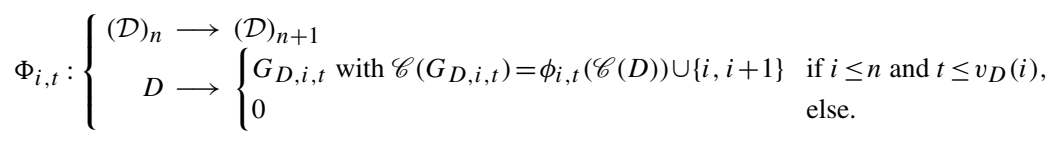

Remarks Let $D$ be a dissection diagram, $i$ be a vertex and $t$ be an integer in $\llbracket 0, v_{D}(i) \rrbracket$. 
1. We easily know the orientation of the chord $\{i, i+1\}$ in $G_{D, i, t}=\Phi_{i, t}(D)$. Indeed, there exists a unique integer $l(i)$ such that the chord labeled by $i$ is the element $a_{D, l(i)}^{i}$ of $A_{D, i}$. If $t \leq l(i)-1$, then $\{i, i+1\}$ is labeled by $i$ else $\{i, i+1\}$ is labeled by $i+1$.

2. The sum of maps $\Phi_{i, t}$ is called operation 1 .

Examples We use the two previous examples by keeping the same color code. The chord inserted is colored in red.

1. For $D=$
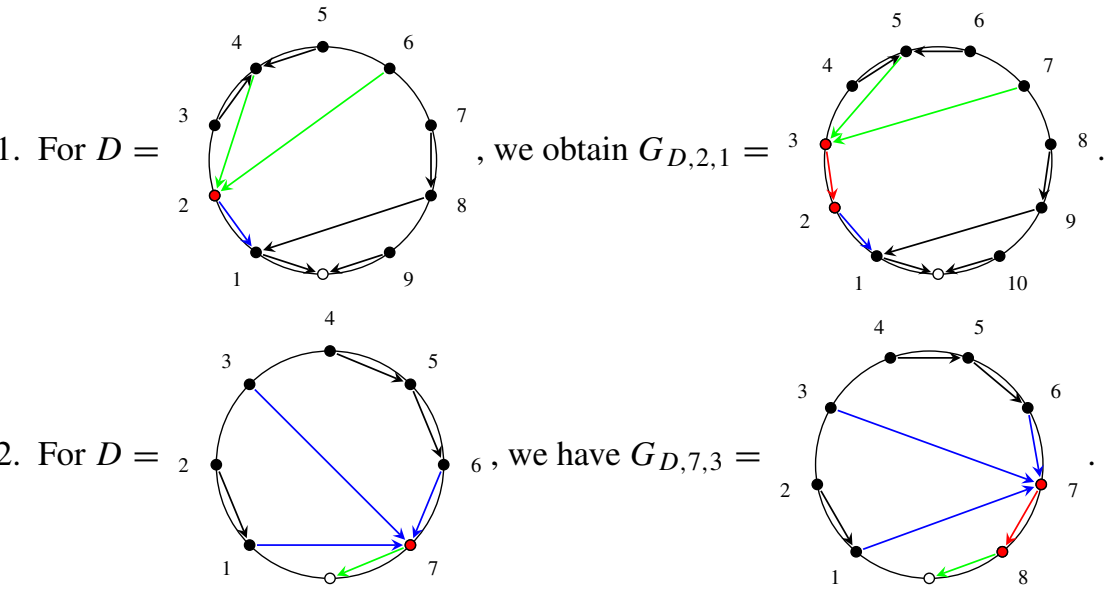

\subsubsection{The chosen vertex of $D$ is its root}

We now consider the root of $D$. Let $\tau \in \llbracket 0, f(0) \rrbracket$ be an integer to split the chords connected with the root. There exists a unique integer $s$ in $\llbracket 1, n \rrbracket$ such that $a_{D, \tau}^{0}=$ $\{0, s\}$. We choose an element $\lambda \in\{0,1\}$ and define two maps.

Case $1 \lambda=0$. The map $\phi_{0, \tau}^{\lambda}$ is defined by:

$$
\phi_{0, \tau}^{\lambda}:\left\{\begin{array}{l}
\mathscr{C}(D) \longrightarrow\{\{u, v\}, 0 \leq u<v \leq n+1\} \\
\{u, v\} \longrightarrow \begin{cases}\{u, v\} & \text { if } u \geq 1 \text { or }(u=0 \text { and } v \leq s-1), \\
\{v, n+1\} & \text { if } u=0 \text { and } v \geq s .\end{cases}
\end{array}\right.
$$

We call $\tilde{G}_{D, 0, \tau}^{\lambda}$ the diagram of degree $n+1$, open between the root and the vertex $n+1$ such that $\mathscr{C}\left(\tilde{G}_{D, 0, \tau}^{\lambda}\right)=\phi_{0, \tau}^{\lambda}(\mathscr{C}(D))$. The map $\phi_{0, \tau}^{\lambda}$ builds an open diagram by creating the vertex $n+1$. 


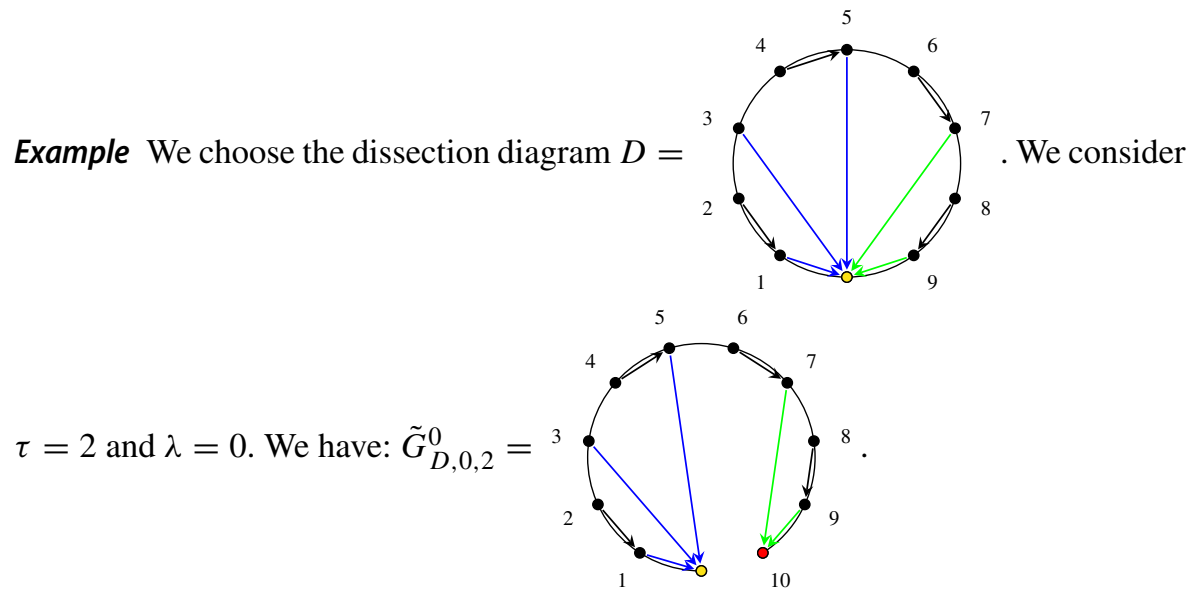

Case $2 \lambda=1$. We define the map $\phi_{0, \tau}^{\lambda}$ by:

$$
\phi_{0, \tau}^{\lambda}:\left\{\begin{array}{l}
\mathscr{C}(D) \longrightarrow\{\{u, v\}, 0 \leq u<v \leq n+1\} \\
\{u, v\} \longrightarrow \begin{cases}\{u+1, v+1\} & \text { if } u \geq 1 \text { or }(u=0 \text { and } v \leq s-1), \\
\{u, v+1\} & \text { if } u=0 \text { and } v \geq s\end{cases}
\end{array}\right.
$$

In this case, the diagram $\tilde{G}_{D, 0, \tau}^{\lambda}$ of degree $n+1$ is open between the root and the vertex 1 and $\mathscr{C}\left(\tilde{G}_{D, 0, \tau}^{\lambda}\right)=\phi_{0, \tau}^{\lambda}(\mathscr{C}(D))$.

Example We use again the dissection diagram $D={ }^{3}$

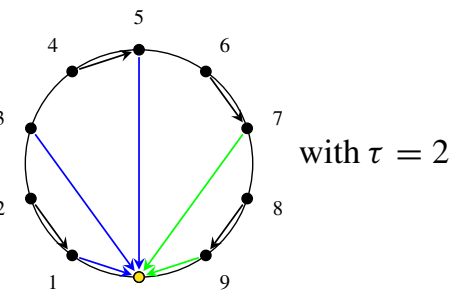

but now $\lambda=1$. We obtain: $\tilde{G}_{D, 0,2}^{1}={ }^{3}$

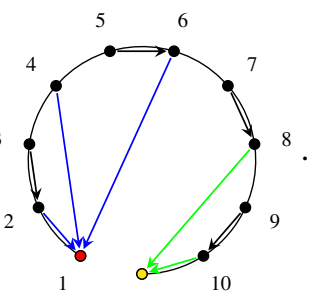

We now define the endomorphism homogeneous of degree 1 coding the insertion of a chord in the root. Therefore, we consider a nonnegative integer (partition of the chords connected with the root) and $\lambda \in\{0,1\}$. We define $\phi_{0, \tau}^{\lambda}$ by:

$$
\Phi_{0, \tau}^{\lambda}:\left\{\begin{aligned}
(\mathcal{D})_{n} & \longrightarrow(\mathcal{D})_{n+1} \\
D & \longrightarrow \begin{cases}G_{0, \tau}^{\lambda}(D) & \text { if } \tau \leq v_{D}(0), \\
0 & \text { else }\end{cases}
\end{aligned}\right.
$$


where the set of the chords of $G_{0, \tau}^{\lambda}(D)$ is:

$$
\mathscr{C}\left(G_{0, \tau}^{\lambda}(D)\right)=\phi_{0, \tau}^{\lambda}(\mathscr{C}(D)) \cup\{\{0,(1-\lambda) n+1\}\}
$$

Remarks Let $D$ be a dissection diagram, $\tau$ be an integer in $\llbracket 0, v_{D}(0) \rrbracket$ and $\lambda$ be an element in $\{0,1\}$.

1. The chord inserted to build $G_{D, 0, \tau}^{\lambda}$ is naturally oriented. Indeed, either $\lambda=0$ and we build $\{0, n+1\}$ (chord labeled by $n+1$ ), or $\lambda=1$ and we build $\{0,1\}$ (chord labeled by 1$)$ in $G_{D, 0, \tau}^{\lambda}$.

2. The sum of all maps $\Phi_{0, t}^{\lambda}$ is called operation 2 .

Examples We use the two previous examples. The inserted chord is colored in red.
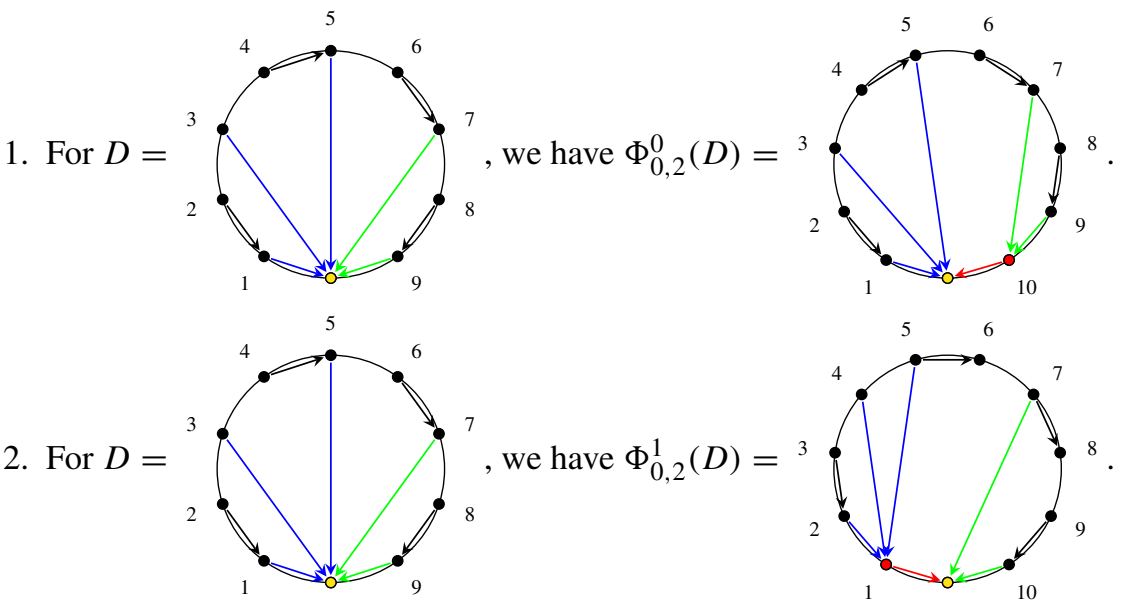

\subsubsection{Computation of $L(D)$ where $D$ is a dissection diagram of degree $n \geq 1$}

Proposition 37 Let $D$ be a dissection diagram of degree $n \geq 1, i$ and $j$ two integers in $\llbracket 1, n \rrbracket, t_{i}$ (respectively $t_{j}$ ) be an integer in $\llbracket 0, f(i) \rrbracket$ (respectively in $\left.\llbracket 0, f(j) \rrbracket\right), \tau_{1}$ and $\tau_{2}$ two integers in $\llbracket 0, f(0) \rrbracket$, and $\lambda_{1}$ and $\lambda_{2}$ two elements in $\{0,1\}$. We have:

1. $\left(\Phi_{i, t_{1}}(D),\{i, i+1\}\right)=\left(\Phi_{j, t_{2}}(D),\{j, j+1\}\right) \Longleftrightarrow\left(i=j\right.$ and $\left.t_{1}=t_{2}\right)$,

2. $\left(\Phi_{0, \tau_{1}}^{\lambda_{1}}(D),\left\{0,\left(1-\lambda_{1}\right) n+1\right\}\right)=\left(\Phi_{0, \tau_{2}}^{\lambda_{2}}(D),\left\{0,\left(1-\lambda_{2}\right) n+1\right\}\right) \Longleftrightarrow\left(\lambda_{1}=\right.$ $\lambda_{2}$ and $\left.\tau_{1}=\tau_{2}\right)$,

3. $\left(\Phi_{i, t_{1}}(D),\{i, i+1\}\right) \neq\left(\Phi_{0, \tau_{1}}^{\lambda_{1}}(D),\left\{0,\left(1-\lambda_{1}\right) n+1\right\}\right)$.

Proof Let $D$ be a dissection diagram of degree $n \geq 1, i$ and $j$ two integers in $\llbracket 1, n \rrbracket$, $t_{i}$ (respectively $t_{j}$ ) be an integer in $\llbracket 0, f(i) \rrbracket$ (respectively in $\left.\llbracket 0, f(j) \rrbracket\right), \tau_{1}$ and $\tau_{2}$ two integers in $\llbracket 0, f(0) \rrbracket$, and $\lambda_{1}$ and $\lambda_{2}$ two elements in $\{0,1\}$.

1. It is sufficient to prove the implication:

$$
\left(\Phi_{i, t_{1}}(D),\{i, i+1\}\right)=\left(\Phi_{i, t_{2}}(D),\{i, i+1\}\right) \Rightarrow t_{1}=t_{2} .
$$


We denote by $p_{1}$ (respectively by $p_{2}$ ) the vertex $i$ of the dissection diagram $\Phi_{i, t_{1}}(D)$ (respectively the dissection diagram $\Phi_{i, t_{2}}(D)$ ), and we consider its valency $v\left(p_{1}\right)$ (respectively $v\left(p_{2}\right)$ ). We obtain $v\left(p_{1}\right)=t_{1}+1$ and $v\left(p_{2}\right)=t_{2}+1$. If we assume that the two couples $\left(\Phi_{i, t_{1}}(D),\{i, i+1\}\right)$ and $\left(\Phi_{i, t_{2}}(D),\{i, i+1\}\right)$ are equal, then the implication becomes trivial.

2. We put $\lambda=\lambda_{1}$. To prove the equivalence, it is sufficient to consider the implication:

$$
\left(\Phi_{0, \tau_{1}}^{\lambda}(D),\{0,(1-\lambda) n+1\}\right)=\left(\Phi_{0, \tau_{2}}^{\lambda}(D),\{0,(1-\lambda) n+1\}\right) \Rightarrow \tau_{1}=\tau_{2} .
$$

We use the same process as before.

3. As $i$ is positive, it is trivial.

Corollary 38 Let $D$ be a dissection diagram of degree $n \geq 1$. We call $\sigma_{D}$ the number of different couples $(G, a)$, where $G$ is a dissection diagram of degree $n+1$ and $a$ is a chord $a=\{u, v\}$ with $0 \leq u<v \leq n+1$, obtained by operations 1 and 2 applied to D. We have:

$$
\sigma_{D}=3 n+2+v_{D}(0)
$$

Proof Let $D$ be a dissection diagram of degree $n \geq 1$. By direct calculation, we have:

$$
\begin{aligned}
\sigma(D) & =\overbrace{\sum_{i=1}^{n}\left(v_{D}(i)+1\right)}^{\text {operation } 1}+\overbrace{2\left(v_{D}(0)+1\right)}^{\text {operation } 2} \\
& =\left(3 n-v_{D}(0)\right)+2\left(v_{D}(0)+1\right) \\
& =3 n+2+v_{D}(0) .
\end{aligned}
$$

Proposition 39 Let $D$ be a dissection diagram of degree $n \in \mathbb{N}^{*}$. We have

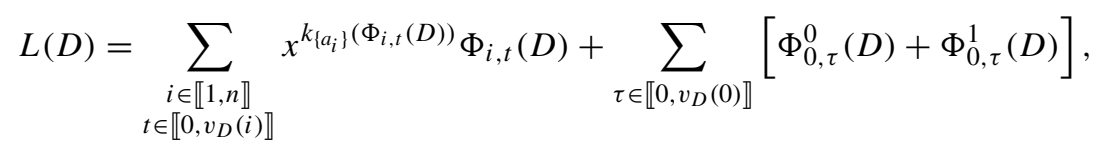

with $a_{i}=\{i, i+1\}$. In other words,

$$
\begin{aligned}
L(D)= & \sum_{\substack{i \in \llbracket 1, n \rrbracket \\
t \in \llbracket 0, l(i)-1 \rrbracket}} \Phi_{i, t}(D)+x \sum_{\substack{i \in \llbracket 1, n \rrbracket \\
t \in \llbracket l(i), v_{D}(i) \rrbracket}} \Phi_{i, t}(D) \\
& +\sum_{\tau \in \llbracket 0, v_{D}(0) \rrbracket}\left[\Phi_{0, \tau}^{0}(D)+\Phi_{0, \tau}^{1}(D)\right]
\end{aligned}
$$

where for any integer $i \in \llbracket 1, n \rrbracket, l(i)$ is the unique integer in $\llbracket 1, v_{D}(i) \rrbracket$ such that the chord $a_{D, l(i)}^{i}$ of $D$ is labeled by $i$. 
Proof The fact that dissection diagrams obtained with operations 1 and 2 are elements of $L(D)$ is trivial.

Let $(G, a) \in(\mathcal{D})_{n+1} \times \mathscr{C}(G)$ such that $q_{\{a\}}=D$. We write $a$ as $a=\{i, j\}$ with $\{i, j\} \in\{\{u, v\}, 0 \leq u<v \leq n+1\}$.

Case $1 i \neq \mathbf{0} q_{\{a\}}(G)=D$ (only one diagram), so $j=i+1$. We have then

$$
(G, a)=\left(\Phi_{i, t}(D),\{i, i+1\}\right) \text { with } t=v_{G}(i)-1 .
$$

Case $2 \boldsymbol{i}=\mathbf{0} q_{\{a\}}(G)=D$ (only one diagram), so $j \in\{1, n+1\}$. We have then

$$
(G, a)= \begin{cases}\left(\Phi_{0, \tau}^{1}(D),\{0,1\}\right) \text { with } \tau=v_{G}(0)-1 & \text { if } j=1 \\ \left(\Phi_{0, \tau}^{0}(D),\{0, n+1\}\right) \text { with } \tau=v_{G}(n+1)-1 & \text { if } j=n+1\end{cases}
$$

By direct calculation, we prove the following corollary.

Corollary 40 We assume $x \in \mathbb{N}$. Let $D$ be a dissection diagram of degree $n \in \mathbb{N}^{*}$. We denote by $\sigma_{L(D)}$ the number of terms in $L(D)$ counted with multiplicity. We have:

$$
\sigma_{L(D)}=3 n x+(1-x) \sum_{i=1}^{n} l(i)+(2-x) v_{D}(0)+2
$$

where for any integer $i \in \llbracket 1, n \rrbracket, l(i)$ is the unique integer in $\llbracket 1, v_{D}(i) \rrbracket$ such that the chord $a_{l(i)}^{i}$ of $D$ is labeled by $i$.

Proposition 41 Let $x$ be a scalar in $\mathbb{K}$. Let $D_{1}$ and $D_{2}$ be two non-empty dissection diagrams. We have the equivalence:

$$
L\left(D_{1}\right)=L\left(D_{2}\right) \Longleftrightarrow D_{1}=D_{2}
$$

Proof Let $x$ be a scalar in $\mathbb{K}$. Let $D_{1}$ and $D_{2}$ be two non-empty dissection diagrams. We recall that $v_{D_{1}}(0)$ (respectively $v_{D_{2}}(0)$ ) is the root valency of $D_{1}$ (respectively $D_{2}$ ). We know that, for any positive integer $n$, the projection of $L\left(D_{1}\right)$ (respectively $L\left(D_{2}\right)$ ) on the linear space of dissection diagrams with the root valency equal to $n$ is positive if $n \in$ $\llbracket 1, v_{D_{1}}(0)+1 \rrbracket \backslash\left\{v_{D_{1}}(0)\right\}$ (respectively $n \in \llbracket 1, v_{D_{2}}(0)+1 \rrbracket \backslash\left\{v_{D_{2}}(0)\right\}$ ), and equals zero if $n \geq v_{D_{1}}(0)+2$ (respectively $\left.n \geq v_{D_{2}}(0)+2\right)$. As a conclusion, if $v_{D_{1}}(0) \neq v_{D_{2}}(0)$ the two elements $L\left(D_{1}\right)$ and $L\left(D_{2}\right)$ are different. We then consider two dissection diagrams $D_{1}$ and $D_{2}$ such that $v_{D_{1}}(0)=v_{D_{2}}(0)$. We assume that $L\left(D_{1}\right)$ and $L\left(D_{2}\right)$

equal. We write $D_{1}$ and $D_{2}$ as $D_{1}=$

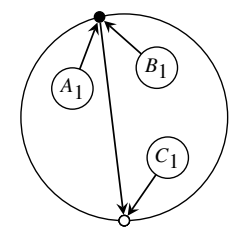
and $D_{2}=$

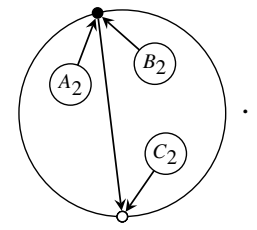



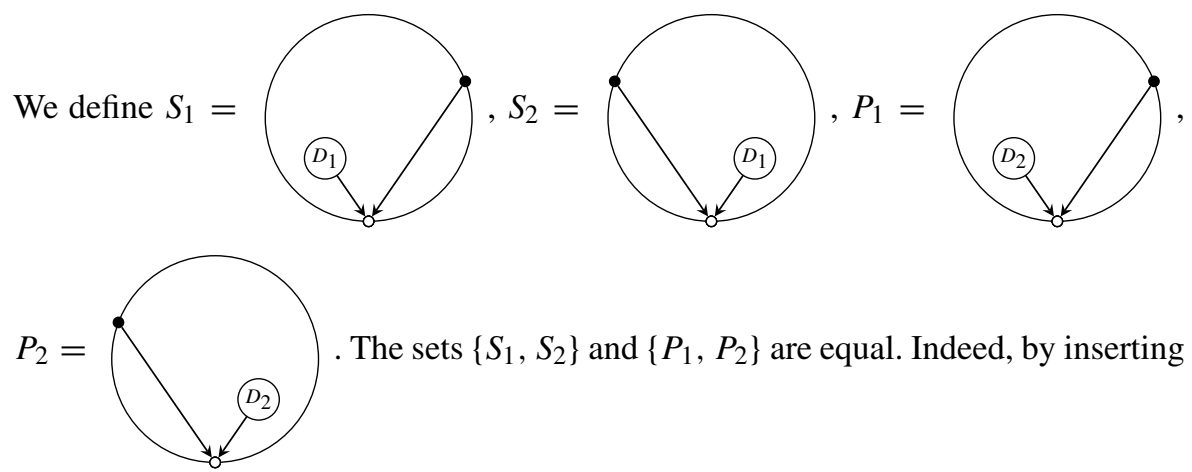

a chord, there exists only two ways to obtain a dissection diagram with a root valency equal to $v_{D_{1}}(0)+1$ (respectively $v_{D_{2}}(0)+1$ ). There are different cases.

If $S_{1}=S_{2}$ or $S_{1} \neq S_{2}$ with $S_{1}=P_{1}$, the result is trivial.

If $S_{1} \neq S_{2}$ and $S_{1}=P_{2}$, then the dissection diagrams $A_{1}, A_{2}, B_{1}, B_{2}$ are all empty.

Furthermore, we have
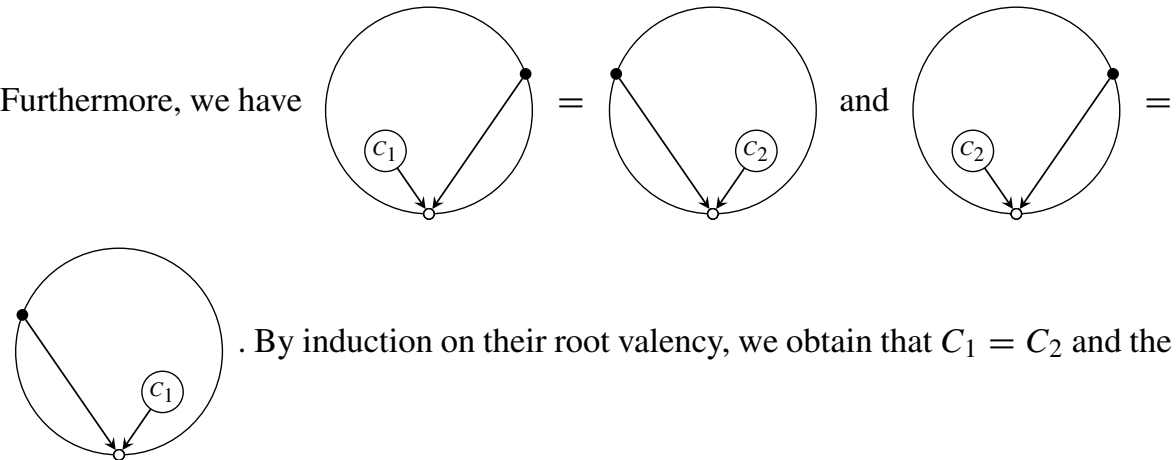

. By induction on their root valency, we obtain that $C_{1}=C_{2}$ and the

proposition is proved.

Proposition 42 Let assume that $\mathbb{R} \subset \mathbb{K}$ and $x \in \mathbb{R}_{+}^{*}$. Let $D$ be a dissection diagram of degree $n$ greater than or equal to 2 . We define the pairing

$$
\langle-,-\rangle:\left\{\begin{aligned}
\mathcal{H}_{\mathrm{D}} \otimes \mathcal{H}_{\mathrm{D}} & \longrightarrow \mathbb{K} \\
D_{1} \ldots D_{k} \otimes G_{1} \ldots G_{l} & \longrightarrow Z_{D_{1} \ldots D_{k}}\left(G_{1} \ldots G_{l}\right) .
\end{aligned}\right.
$$

Then for any dissection diagram $D$ of degree $n \geq 2$, there exists a dissection diagram $G$ of degree $n-1$ such that $\langle D, L(G)\rangle \neq 0$.

Proof Let $x$ be a positive scalar and $D$ a dissection diagram of degree $n$ greater than or equal to 2 . As there is not any intersection between its chords, there exists a chord $a$ such that $a$ connects two consecutive vertices. We call $G$ the dissection diagram $G=q_{\{a\}}(D)$. It has a degree equal to $n-1$, which establishes the proposition.

Proposition 43 Let assume that $\mathbb{R} \subset \mathbb{K}$ and $x \in \mathbb{R}_{+}^{*}$. Let $n$ be a nonnegative integer and $e_{n} \in \mathcal{T}_{\mathrm{R}}$ the ladder of degree $n$. By using the previous pairing, we have that for any nonnegative integer $n$ and any dissection diagram $D$ of degree $n$ the scalar $\left\langle D, \varphi\left(e_{n}\right)\right\rangle$ is positive. 
Proof We give a recursive proof. The result is trivial for $e_{1}$. We assume the result true for a particular positive rank $n$. We write $\varphi\left(e_{n}\right)=\sum_{\substack{G \in(\mathcal{D})_{n} \\ \text { diagram }}} a_{G} G$ where each $a_{G}$ is positive. Then,

$$
\varphi\left(e_{n+1}\right)=\varphi\left(e_{n}\right) \triangleright \bigcirc=\sum_{\substack{G \in(\mathcal{D})_{n} \\ \text { diagram }}} a_{G} L(G)=\sum_{\substack{D \in(\mathcal{D})_{n+1} \\ \text { diagram }}} b_{D} D
$$

and, according to the previous proposition, all $b_{D}$ are positive scalars. The result is true for the rank $n+1$, so the proposition is proved.

\subsection{Insertion of a chord in two dissection diagrams}

Let $D_{1}$ and $D_{2}$ be two dissection diagrams of positive degree $n_{1}$ and $n_{2}$. We choose a vertex $i$ of $D_{1}$, a vertex $j$ of $D_{2}$, integers $t \in \llbracket 0, v_{D_{1}}(i) \rrbracket$ and $\tau \in \llbracket 0, v_{D_{2}}(j) \rrbracket$. We insert a new chord by using the following steps. Thanks to the opening maps defined in Sect. 3.1, we obtain two open diagrams. Then, we change the vertices labels and insert a new chord to have a dissection diagram of degree $n_{1}+n_{2}+1$. Since a dissection diagram has only one root, we open at least one of the two dissection diagrams $D_{1}$ and $D_{2}$ at the root. We introduce an integer $\lambda \in\{0,1\}$ that will be useful in the sequel.

\subsubsection{Open $D_{1}$ at the root and $D_{2}$ at another vertex}

We consider the root of $D_{1}$, an integer $t \in \llbracket 0, v_{D_{1}}(0) \rrbracket$ (partition of the chords of $D_{1}$ connected with the root), an integer $\lambda \in\{0,1\}$ (location of the root of the open diagram $\tilde{G}_{0, t}^{\lambda}$ obtained with $D_{1}$ ), a vertex of $D_{2}$ different from the root (i.e. an integer $j \in \llbracket 1, n_{2} \rrbracket$ ) and an integer $\tau \in \llbracket 0, v_{D_{2}}(j) \rrbracket$ (partition of the chords of $D_{2}$ with the vertex $j$ in common). The root of the dissection diagram $G_{D_{1}, D_{2}, t, j, \tau}^{\lambda}$ built after the insertion is given by the root of $D_{2}$.

According to paragraph 3.1, thanks to the map $\phi_{0, t}^{\lambda}$ (respectively $\phi_{j, \tau}$ ), we can consider the open diagram $\tilde{G}_{D_{1}, 0, t}^{\lambda}$ equipped with the set $\phi_{0, t}^{\lambda}\left(\mathscr{C}\left(D_{1}\right)\right)$ (respectively $\tilde{G}_{D_{2}, j, \tau}$ equipped with the set $\left.\phi_{j, \tau}\left(\mathscr{C}\left(D_{2}\right)\right)\right)$.

To change the labels of the chords, as the root of $G_{D_{1}, D_{2}, t, j, \tau}^{\lambda}$ originates from the root of $D_{2}$, we consider the maps:

$$
\gamma_{D_{1}, t}^{\lambda}:\left\{\begin{aligned}
\phi_{t, \lambda}^{0}\left(\mathscr{C}\left(G_{1}\right)\right) & \longrightarrow\left\{\{u, v\}, 0 \leq u<v \leq n_{1}+n_{2}+1\right\} \\
\{u, v\} & \longrightarrow \begin{cases}\{u+j-\lambda, v+j-\lambda\} & \text { if } u \geq 1, \\
\left\{j+\lambda\left(n_{1}+1\right), v+j-\lambda\right\} & \text { if } u=0\end{cases}
\end{aligned}\right.
$$


and

$$
\gamma_{D_{2}, j, \tau}:\left\{\begin{aligned}
\phi_{j, \tau}\left(\mathscr{C}\left(G_{2}\right)\right) & \longrightarrow\left\{\{u, v\}, 0 \leq u<v \leq n_{1}+n_{2}+1\right\} \\
\{u, v\} & \longrightarrow \begin{cases}\{u, v\} & \text { if } u \leq j \text { and } v \leq j, \\
\left\{u, v+n_{1}\right\} & \text { if } u \leq j \text { and } v \geq j+1, \\
\left\{u+n_{1}, v+n_{1}\right\} & \text { if } u \geq j+1 .\end{cases}
\end{aligned}\right.
$$

With these functions, we have a diagram of degree $n_{1}+n_{2}+1$ which is not a dissection diagram since there are only $n_{1}+n_{2}$ chords. We just have to build the chord $\{j, j+$ $\left.n_{1}+1\right\}$.

We now define a morphism from $\mathcal{D} \otimes \mathcal{D}$ to $\mathcal{D}$ homogeneous of degree 1 . We consider two nonnegative integers $t$ and $\tau$, an element $\lambda$ in $\{0,1\}$, a positive integer $j$ and the following map:

$\Gamma_{t, j, \tau}^{\lambda}:\left\{\begin{aligned}(\mathcal{D})_{n_{1}} \otimes(\mathcal{D})_{n_{2}} & \longrightarrow(\mathcal{D})_{n_{1}+n_{2}+1} \\ D_{1} \otimes D_{2} & \longrightarrow \begin{cases}G_{D_{1}, D_{2}, t, j, \tau}^{\lambda} & \text { if } t \leq v_{D_{1}}(0), j \leq n_{2} \text { and } \tau \leq v_{D_{2}}(j), \\ 0 & \text { else, }\end{cases} \end{aligned}\right.$

where the set $\mathscr{C}\left(G_{D_{1}, D_{2}, t, j, \tau}^{\lambda}\right)$ is given by:

$\mathscr{C}\left(G_{D_{1}, D_{2}, t, j, \tau}^{\lambda}\right)=\gamma_{D_{1}, t}^{\lambda}\left(\phi_{0, t}^{\lambda}\left(\mathscr{C}\left(D_{1}\right)\right)\right) \cup \gamma_{D_{2}, j, \tau}\left(\phi_{j, \tau}\left(\mathscr{C}\left(G_{2}\right)\right)\right) \cup\left\{\left\{j, j+n_{1}+1\right\}\right\}$.

Example We use the dissection diagrams $D_{1}={ }_{3}$ and $D_{2}=$ with $\lambda=1, t=1, j=2$ and $\tau=1$. We first build $\tilde{G}_{D_{1}, 0,1}^{1}$ and

$\tilde{G}_{D_{2}, 2,1}$ by keeping the color code explained in Sect. 3.1 . Finally, we make the insertion. The old root of $D_{1}$ becomes white, and the new chord is colored in red. We have: 


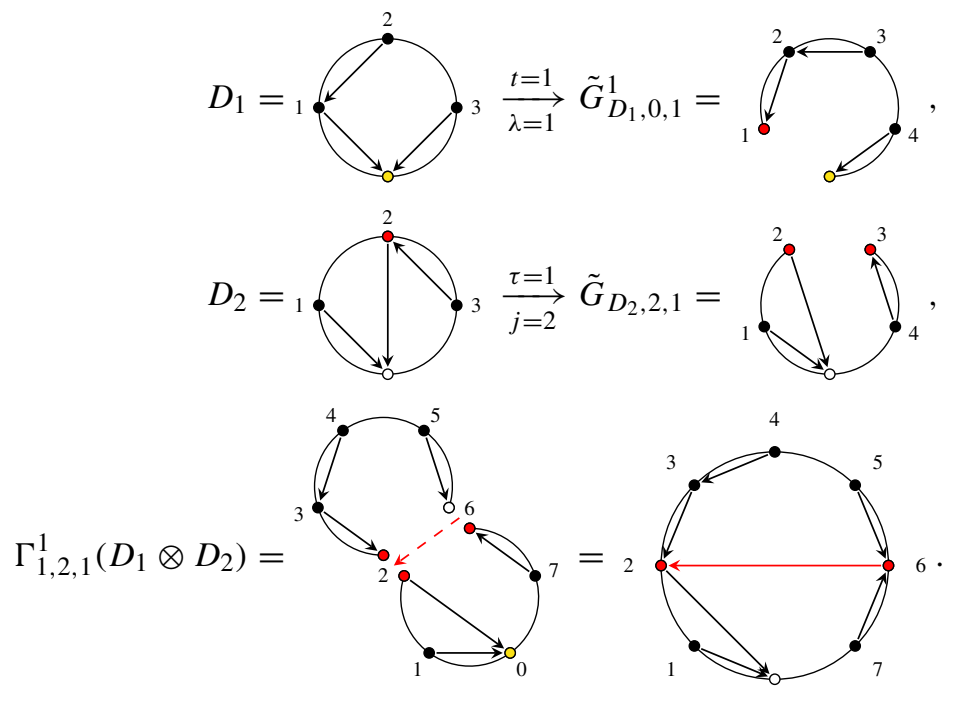

Remarks Let $D_{1}$ and $D_{2}$ be two dissection diagrams of respective positive degree $n_{1}$ and $n_{2}$ and $\lambda \in\{0,1\}, t \in \llbracket 0, v_{D_{1}}(0) \rrbracket, j \in \llbracket 1, n_{2} \rrbracket$ and $\tau \in \llbracket 0, v_{D_{2}}(j) \rrbracket$ be integers.

1. It is not necessary to consider the two possible values of $\lambda$. Indeed, by direct calculation we have $\Gamma_{t, j, \tau}^{\lambda}\left(D_{1} \otimes D_{2}\right)=\Gamma_{t, j, \tau}^{1-\lambda}\left(D_{1} \otimes D_{2}\right)$.

2. We easily know the orientation of the inserted chord $\left\{j, j+n_{1}+1\right\}$. Indeed, there exists a unique integer $l(j)$ such that the chord $a_{D_{2}, l(j)}^{j}$ of $D_{2}$ is labeled by $j$. If $\tau \leq l(j)-1$, then the chord $\left\{j, j+n_{1}+1\right\}$ of $\Gamma_{t, j, \tau}^{\lambda}\left(D_{1} \otimes D_{2}\right)$ is labeled by $j$ else $\tau \geq l(j)$ and the chord $\left\{j, j+n_{1}+1\right\}$ of $\Gamma_{t, j, \tau}^{\lambda}\left(D_{1} \otimes D_{2}\right)$ is labeled by $j+n_{1}+1$.

3. We call $\kappa$ the map sending $D_{1} \otimes D_{2}$ to $D_{2} \otimes D_{1}$. The sum of maps of types $\Gamma_{t, j, \tau}^{\lambda}$ or $\Gamma_{t, j, \tau}^{\lambda} \circ \kappa$ is called operation 3 .

\subsubsection{Open $D_{1}$ and $D_{2}$ at the root}

We now work with the root of the two dissection diagrams. We consider an integer $t \in \llbracket 0, v_{D_{1}}(0) \rrbracket$ (partition of the chords of $D_{1}$ connected with the root), an integer $\tau \in \llbracket 0, v_{D_{2}}(0) \rrbracket$ (partition of the chords of $D_{2}$ connected with the root) and two integers $\lambda_{1}, \lambda_{2} \in\{0,1\}$ (location of the root of the two diagrams $\tilde{G}_{D_{1}, 0, t}^{\lambda_{1}}$ and $\tilde{G}_{D_{2}, 0, \tau}^{\lambda_{2}}$, built with the opening maps defined in Sect. 3.1). The root of the dissection diagram $G_{D_{1}, D_{2}, t, \tau}^{\lambda_{1}, \lambda_{2}}$, built after insertion, is again given by the root of $D_{2}$. There are two cases.

Case $1 \lambda=\lambda_{2}=0$ and $\lambda_{1}=1-\lambda=1$. According to paragraph 3.1, thanks to the map $\phi_{0, t}^{1}$ (respectively $\phi_{0, \tau}^{0}$ ), we can consider the open diagram $\tilde{G}_{D_{1}, 0, t}^{1}$ equipped with the set $\phi_{0, t}^{1}\left(\mathscr{C}\left(D_{1}\right)\right)$ (respectively $\tilde{G}_{D_{2}, 0, \tau}^{0}$ with the set $\phi_{0, \tau}^{0}\left(\mathscr{C}\left(D_{2}\right)\right)$ ).

To change labels, as the root of $G_{D_{1}, D_{2}, t, \tau}^{1,1}$ is given by the root of $D_{2}$, we use the following maps: 


$$
\gamma_{D_{1}, t}^{1}:\left\{\begin{array}{c}
\phi_{0, t}^{1}\left(\mathscr{C}\left(D_{1}\right)\right) \\
\{u, v\} \rightarrow \begin{cases}\left\{u+n_{2}, v+n_{2}\right\} & \text { if } u \geq 1 \\
\left\{u, v+n_{2}\right\} & \text { if } u=0\end{cases}
\end{array}\right.
$$

and

$$
\gamma_{D_{2}, \tau}^{0}:\left\{\begin{aligned}
\phi_{0, \tau}^{0}\left(\mathscr{C}\left(D_{2}\right)\right) & \longrightarrow\left\{\{u, v\}, 0 \leq u<v \leq n_{1}+n_{2}+1\right\} \\
\{u, v\} & \longrightarrow\{u, v\} .
\end{aligned}\right.
$$

The diagram of degree $n_{1}+n_{2}+1$ built with the previous map is not a dissection diagram since there are just $n_{1}+n_{2}$ chords. We need only add the chord $\left\{0, n_{2}+1\right\}$.

We define a morphism from $\mathcal{D} \otimes \mathcal{D}$ to $\mathcal{D}$ homogeneous of degree 1 . Let $t$ and $\tau$ be two nonnegative integers and we consider the map:

$$
\Gamma_{t, \tau}^{0}:\left\{\begin{aligned}
(\mathcal{D})_{n_{1}} \otimes(\mathcal{D})_{n_{2}} & \longrightarrow(\mathcal{D})_{n_{1}+n_{2}+1} \\
D_{1} \otimes D_{2} & \longrightarrow \begin{cases}G_{D_{1}, D_{2}, t, \tau}^{1,0} & \text { si } t \leq v_{D_{1}}(0) \text { and } \tau \leq v_{D_{2}}(0), \\
0 & \text { sinon, }\end{cases}
\end{aligned}\right.
$$

with

$$
\mathscr{C}\left(G_{D_{1}, D_{2}, t, \tau}^{1,0}\right)=\gamma_{D_{1}, t}^{1}\left(\phi_{0, t}^{1}\left(\mathscr{C}\left(D_{1}\right)\right)\right) \cup \gamma_{D_{2}, \tau}^{0}\left(\phi_{0, \tau}^{0}\left(\mathscr{C}\left(D_{2}\right)\right)\right) \cup\left\{\left\{0, n_{2}+1\right\}\right\} .
$$

Example We use the dissection diagrams $D_{1}=$

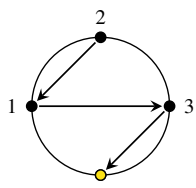
and $D_{2}=$
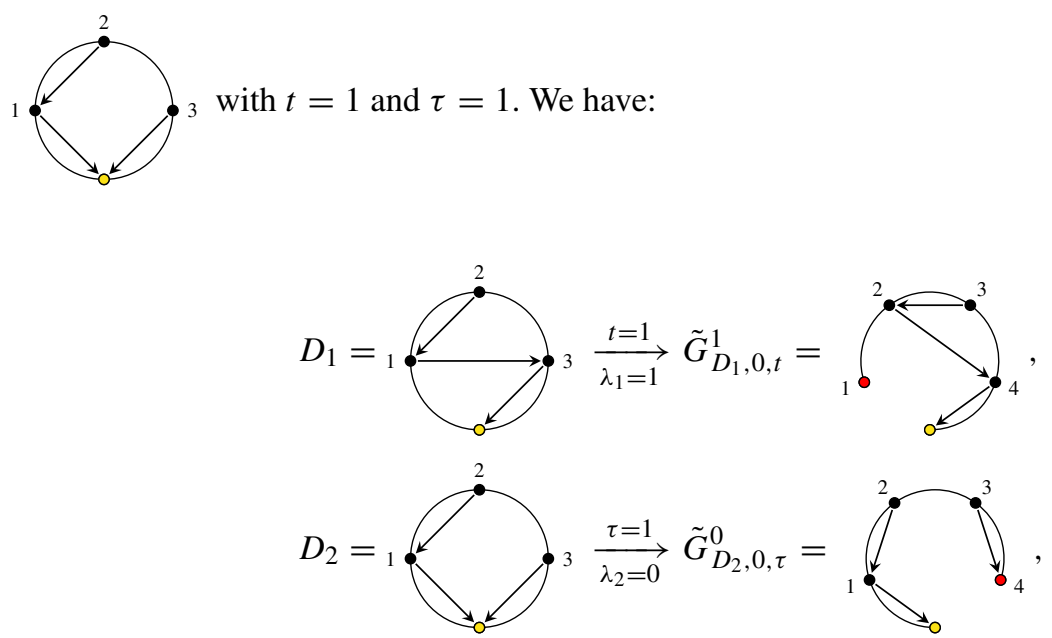


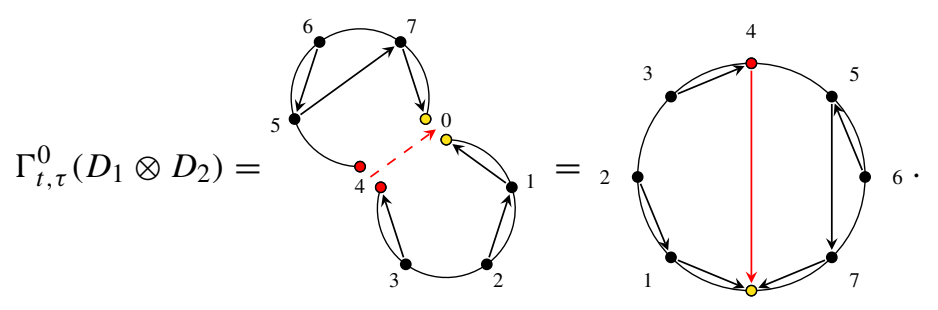

Case $2 \lambda=\lambda_{2}=1$ and $\lambda_{1}=1-\lambda=0$. We now consider the open diagram $\tilde{G}_{D_{1}, 0, t}^{0}$ equipped with the set $\phi_{0, t}^{0}\left(\mathscr{C}\left(D_{1}\right)\right)$ (respectively $\tilde{G}_{D_{2}, 0, \tau}^{1}$ with the set $\phi_{0, \tau}^{1}\left(\mathscr{C}\left(D_{2}\right)\right)$ ).

To change labels, we use:

$$
\gamma_{D_{1}, t}^{0}:\left\{\begin{aligned}
\phi_{0, t}^{0}\left(\mathscr{C}\left(D_{1}\right)\right) & \longrightarrow\left\{\{u, v\}, 0 \leq u<v \leq n_{1}+n_{2}+1\right\} \\
\{u, v\} & \longrightarrow\{u, v\}
\end{aligned}\right.
$$

and

$$
\gamma_{D_{2}, \tau}^{1}:\left\{\begin{aligned}
\phi_{0, \tau}^{1}\left(\mathscr{C}\left(D_{2}\right)\right) & \longrightarrow\left\{\{u, v\}, 0 \leq u<v \leq n_{1}+n_{2}+1\right\} \\
\{u, v\} & \longrightarrow \begin{cases}\left\{u+n_{1}, v+n_{1}\right\} & \text { if } u \geq 1 \\
\left\{u, v+n_{1}\right\} & \text { if } u=0\end{cases}
\end{aligned}\right.
$$

To have a dissection diagram of degree $n_{1}+n_{2}+1$, we add the chord $\left\{0, n_{1}+1\right\}$.

Let $t$ and $\tau$ be two nonnegative integers and we define the morphism $\Gamma_{t, \tau}^{1}$ homogeneous of degree 1 by:

$$
\Gamma_{t, \tau}^{1}:\left\{\begin{aligned}
(\mathcal{D})_{n_{1}} \otimes(\mathcal{D})_{n_{2}} & \longrightarrow(\mathcal{D})_{n_{1}+n_{2}+1} \\
D_{1} \otimes D_{2} & \longrightarrow \begin{cases}G_{D_{1}, D_{2}, t, \tau}^{0,1} & \text { if } t \leq v_{D_{1}}(0) \text { and } \tau \leq v_{D_{2}}(0), \\
0 & \text { else, }\end{cases}
\end{aligned}\right.
$$

with

$$
\mathscr{C}\left(G_{D_{1}, D_{2}, t, \tau}^{0,1}\right)=\gamma_{D_{1}, t}^{0}\left(\phi_{0, t}^{0}\left(\mathscr{C}\left(D_{1}\right)\right)\right) \cup \gamma_{D_{2}, \tau}^{1}\left(\phi_{0, \tau}^{1}\left(\mathscr{C}\left(D_{2}\right)\right)\right) \cup\left\{\left\{0, n_{1}+1\right\}\right\} .
$$

Example With $D_{1}=1$ have:

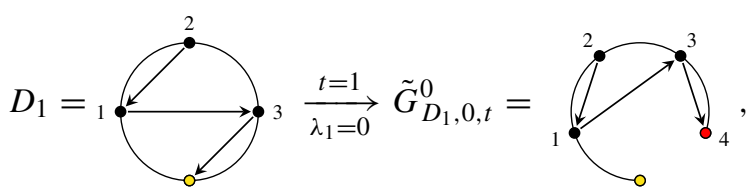




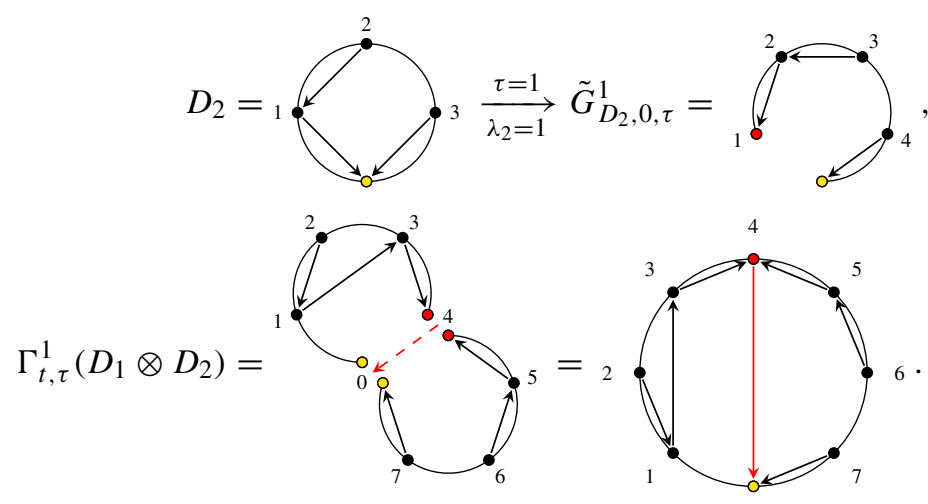

Remarks Let $D_{1}$ and $D_{2}$ be two dissection diagrams of respective positive degree $n_{1}$ and $n_{2}$ and $\lambda_{1}, \lambda_{2} \in\{0,1\}, t \in \llbracket 0, v_{D_{1}}(0) \rrbracket$ and $\tau \in \llbracket 0, v_{D_{2}}(0) \rrbracket$ be integers.

1. By direct calculation, we have:

$$
\Gamma_{t, \tau}^{0}\left(D_{1} \otimes D_{2}\right)=\Gamma_{\tau, t}^{1}\left(D_{2} \otimes D_{1}\right) \text { and } \Gamma_{t, \tau}^{1}\left(D_{1} \otimes D_{2}\right)=\Gamma_{\tau, t}^{0}\left(D_{2} \otimes D_{1}\right) .
$$

2. The two cases $\left(\lambda_{1}=0, \lambda_{2}=0\right)$ and $\left(\lambda_{1}=1, \lambda_{2}=1\right)$ can be ignored. Indeed, it is sufficient to use the two maps

$$
\tilde{\gamma}_{D_{1}, t}^{0}:\left\{\begin{aligned}
\phi_{0, t}^{0}\left(\mathscr{C}\left(D_{1}\right)\right) & \longrightarrow\left\{\{u, v\}, 0 \leq u<v \leq n_{1}+n_{2}+1\right\} \\
\{u, v\} & \longrightarrow \begin{cases}\left\{u+n_{2}+1, v+n_{2}+1\right\} & \text { si } v \leq n_{1} \\
\left\{0, u+n_{2}+1\right\} & \text { si } v=n_{1}+1\end{cases}
\end{aligned}\right.
$$

and

$$
\tilde{\gamma}_{D_{1}, t}^{1}:\left\{\begin{aligned}
\phi_{0, t}^{1}\left(\mathscr{C}\left(D_{1}\right)\right) & \longrightarrow\left\{\{u, v\}, 0 \leq u<v \leq n_{1}+n_{2}+1\right\} \\
\{u, v\} & \longrightarrow \begin{cases}\{u-1, v-1\} & \text { si } u \geq 1, \\
\left\{v-1, n_{1}+1\right\} & \text { si } u=0,\end{cases}
\end{aligned}\right.
$$

and the dissection diagrams $G_{D_{1}, D_{2}, t, \tau}^{0,0}$ and $G_{D_{1}, D_{2}, t, \tau}^{1,1}$ of degree $n_{1}+n_{2}+1$ with

$$
\begin{aligned}
& \mathscr{C}\left(G_{D_{1}, D_{2}, t, \tau}^{0,0}\right)=\tilde{\gamma}_{D_{1}, t}^{0}\left(\phi_{0, t}^{0}\left(\mathscr{C}\left(D_{1}\right)\right)\right) \cup \gamma_{D_{2}, \tau}^{0}\left(\phi_{0, \tau}^{0}\left(\mathscr{C}\left(D_{2}\right)\right)\right) \cup\left\{\left\{0, n_{2}+1\right\}\right\}, \\
& \mathscr{C}\left(G_{D_{1}, D_{2}, t, \tau}^{1,1}\right)=\tilde{\gamma}_{D_{1}, t}^{1}\left(\phi_{0, t}^{1}\left(\mathscr{C}\left(D_{1}\right)\right)\right) \cup \gamma_{D_{2}, \tau}^{1}\left(\phi_{0, \tau}^{1}\left(\mathscr{C}\left(D_{2}\right)\right)\right) \cup\left\{\left\{0, n_{1}+1\right\}\right\} .
\end{aligned}
$$

Then we have: $G_{D_{1}, D_{2}, t, \tau}^{0,0}=G_{D_{1}, D_{2}, t, \tau}^{1,0}$ and $G_{D_{1}, D_{2}, t, \tau}^{1,1}=G_{D_{1}, D_{2}, t, \tau}^{0,1}$.

3. The sum of maps of type $\Gamma_{t, \tau}^{\lambda}$ is called operation 4 .

\subsubsection{Computation of $L\left(D_{1} D_{2}\right)$ where $\left(D_{1}, D_{2}\right) \in(\mathcal{D})_{n_{1}} \times(\mathcal{D})_{n_{2}}$ and $n_{1}, n_{2} \geq 1$}

Proposition 44 Let $D_{1}$ and $D_{2}$ be two dissection diagrams of positive degree $n_{1}$ and $n_{2}, i$ in $\llbracket 1, n_{1} \rrbracket$, $\varrho$ in $\llbracket 0, v_{D_{1}}(i) \rrbracket, p$ in $\llbracket 0, v_{D_{2}}(0) \rrbracket, j, j_{1}$ and $j_{2}$ in $\llbracket 1, n_{2} \rrbracket, \tau$, respectively 
$\tau_{1}$, respectively $\tau_{2}$ in $\llbracket 0, v_{D_{2}}(j) \rrbracket$, respectively $\llbracket 0, v_{D_{2}}\left(j_{1}\right) \rrbracket$, respectively $\llbracket 0, v_{D_{2}}\left(j_{2}\right) \rrbracket$, $\omega, \omega_{1}$ and $\omega_{2}$ in $\llbracket 0, v_{D_{2}}(0) \rrbracket, t, t_{1}$ and $t_{2}$ in $\left.\llbracket 0, v_{D_{1}}(0) \rrbracket\right)$ in $\lambda, \lambda_{1}, \lambda_{2}$ in $\{0,1\}$ be integers.

We assume first that $D_{1}$ and $D_{2}$ are two different dissection diagrams. We have the following statements:

1. $\left(\Gamma_{t_{1}, j_{1}, \tau_{1}}^{1}\left(D_{1} \otimes D_{2}\right),\left\{j_{1}, j_{1}+n_{1}+1\right\}\right)=\left(\Gamma_{t_{2}, j_{2}, \tau_{2}}^{1}\left(D_{1} \otimes D_{2}\right),\left\{j_{2}, j_{2}+n_{1}+1\right\}\right)$ is equivalent to $\left(j_{1}=j_{2}, t_{1}=t_{2}\right.$ and $\left.\tau_{1}=\tau_{2}\right)$,

2. $\left(\Gamma_{t_{1}, \omega_{1}}^{\lambda_{1}}\left(D_{1} \otimes D_{2}\right),\left\{0, \lambda_{1} n_{1}+\left(1-\lambda_{1}\right) n_{2}+1\right\}\right)=\left(\Gamma_{t_{2}, \omega_{2}}^{\lambda_{2}}\left(D_{1} \otimes D_{2}\right),\left\{0, \lambda_{2} n_{1}\right.\right.$ $\left.\left.+\left(1-\lambda_{2}\right) n_{2}+1\right\}\right)$ is equivalent to $\left(t_{1}=t_{2}, \omega_{1}=\omega_{2}\right.$ and $\left.\lambda_{1}=\lambda_{2}\right)$,

3. $\left(\Gamma_{t_{1}, j_{1}, \tau_{1}}^{1}\left(D_{1} \otimes D_{2}\right),\left\{j, j+n_{1}+1\right\}\right) \neq\left(\Gamma_{t_{2}, \omega_{2}}^{\lambda}\left(D_{1} \otimes D_{2}\right),\left\{0, \lambda_{2} n_{1}+\left(1-\lambda_{2}\right) n_{2}\right.\right.$ $+1\})$,

4. $\left(\Gamma_{t, j, \tau}^{1}\left(D_{1} \otimes D_{2}\right),\left\{j, j+n_{1}+1\right\}\right) \neq\left(\Gamma_{p, i, \varrho}^{1}\left(D_{2} \otimes D_{1}\right),\left\{i, i+n_{2}+1\right\}\right)$.

We assume that $D_{1}$ equals $D_{2}$, and we denote $D=D_{1}=D_{2}$. We have the following statements:

5. $\left(\Gamma_{t_{1}, j_{1}, \tau_{1}}^{1}(D \otimes D),\left\{j_{1}, j_{1}+n_{1}+1\right\}\right)=\left(\Gamma_{t_{2}, j_{2}, \tau_{2}}^{1}(D \otimes D),\left\{j_{2}, j_{2}+n_{1}+1\right\}\right)$ is equivalent to $\left(j_{1}=j_{2}, t_{1}=t_{2}\right.$ and $\left.\tau_{1}=\tau_{2}\right)$,

6. $\left(\Gamma_{t, \omega}^{\lambda}(D \otimes D),\left\{0, n_{1}+1\right\}\right)=\left(\Gamma_{\omega, t}^{1-\lambda}(D \otimes D),\left\{0, n_{1}+1\right\}\right)$,

7. $\left(\Gamma_{t_{1}, j_{1}, \tau_{1}}^{1}(D \otimes D),\left\{j, j+n_{1}+1\right\}\right) \neq\left(\Gamma_{t_{2}, \omega_{2}}^{\lambda}(D \otimes D),\left\{0, n_{1}+1\right\}\right)$.

Proof We assume that $D_{1}$ and $D_{2}$ are two different dissection diagrams.

1. It is sufficient to prove:

$$
\Gamma_{t_{1}, j, \tau_{1}}^{1}\left(D_{1} \otimes D_{2}\right)=\Gamma_{t_{2}, j, \tau_{2}}^{1}\left(D_{1} \otimes D_{2}\right) \Longrightarrow\left(t_{1}=t_{2} \text { and } \tau_{1}=\tau_{2}\right)
$$

We assume $K_{1}=\Gamma_{t_{1}, j, \tau_{1}}^{1}\left(D_{1} \otimes D_{2}\right)$ equals $K_{2}=\Gamma_{t_{2}, j, \tau_{2}}^{1}\left(D_{1} \otimes D_{2}\right)$. We denote by $p_{1}(j)$ (respectively by $p_{2}(j)$ ) the vertex $j$ of the dissection diagram $K_{1}$ (respectively of the dissection diagram $K_{2}$ ), and we consider its valency $v_{1}(j)$ (respectively $v_{2}(j)$ ). We have:

$$
v_{1}(j)=v_{D_{1}}(0)-t_{1}+\tau_{1}+1=v_{D_{1}}(0)-t_{2}+\tau_{2}+1=v_{2}(j)
$$

We now use the subset of chords $A_{K_{1}, j}$ (respectively $A_{K_{2}, j}$ ) and the chord $a=$ $\left\{j, j+n_{1}+1\right\}$ too. We know that $a=a_{K_{1}, \tau_{1}+1}^{j}=a_{K_{2}, \tau_{2}+1}^{j}$, so we have $t_{1}=t_{2}$ and $\tau_{1}=\tau_{2}$.

2. We assume that the two dissection diagrams $\Gamma_{t_{1}, \omega_{1}}^{\lambda_{1}}\left(D_{1} \otimes D_{2}\right)$ and $\Gamma_{t_{2}, \omega_{2}}^{\lambda_{2}}\left(D_{1} \otimes D_{2}\right)$ are equal. We further assume that the two chords $\left\{0, \lambda_{1} n_{1}+\left(1-\lambda_{1}\right) n_{2}+1\right\}$ and $\left\{0, \lambda_{2} n_{1}+\left(1-\lambda_{2}\right) n_{2}+1\right\}$ are equal and $\lambda_{1} \neq \lambda_{2}$. We then obtain $n_{1}=$ $n_{2}$. Without loss of generality, we can use the case $\lambda_{1}=0$ and $\lambda_{2}=1$. We denote $K_{1}=\Gamma_{t_{1}, \omega_{1}}^{\lambda_{1}}\left(D_{1} \otimes D_{2}\right)$ and $K_{2}=\Gamma_{t_{2}, \omega_{2}}^{\lambda_{2}}\left(D_{1} \otimes D_{2}\right)$, and consider the subgraph of $K_{1}$ (respectively the subgraph of $K_{2}$ ) created with vertices from the set $\left\{1, \ldots, n_{1}+1\right\}$ and contract $\left\{0, n_{1}+1\right\}$. Since $K_{1}$ and $K_{2}$ are equal, the resulting dissection diagrams are equal as well. And yet, with $K_{1}$ (respectively $K_{2}$ ) we obtain $D_{2}$ (respectively $D_{1}$ ) and thus $D_{1}=D_{2}$. We can conclude that $\lambda_{1}$ and $\lambda_{2}$ are equal. Without loss of generality, we assume $\lambda_{1}=\lambda_{2}=0$. Let $p_{1}$ 
(respectively $p_{2}$ ) be the root of $K_{1}$ (respectively $K_{2}$ ). We consider their valency $v_{1}$ (respectively $v_{2}$ ). We have:

$$
v_{1}=v_{D_{2}}(0)-\omega_{1}+t_{1}+1=v_{D_{2}}(0)-\omega_{2}+t_{2}+1=v_{2} .
$$

We now use the subset of chords $A_{K_{1}, 0}$ (respectively $A_{K_{2}, 0}$ ) and the chord $a=$ $\left\{0, n_{2}+1\right\}$. We have $a=a_{K_{1}, t_{1}+1}^{0}=a_{K_{2}, t_{2}+1}^{0}$, so $t_{1}=t_{2}$ and $\omega_{1}=\omega_{2}$.

3. As $j$ is positive, this point is trivial.

4. We assume there exists integer $j \in \llbracket 1, n_{2} \rrbracket$ (respectively $i \in \llbracket 1, n_{1} \rrbracket$ ), $t \in$ $\llbracket 0, v_{D_{1}}(0) \rrbracket$ and $\tau \in \llbracket 0, v_{D_{2}}(j) \rrbracket$ (respectively $p \in \llbracket 0, v_{D_{2}}(0) \rrbracket$ and $\varrho \in$ $\left.\llbracket 0, v_{D_{1}}(i) \rrbracket\right)$ such that

$$
\left(\Gamma_{t, j, \tau}^{1}\left(D_{1} \otimes D_{2}\right),\left\{j, j+n_{1}+1\right\}\right)=\left(\Gamma_{p, i, \varrho}^{1}\left(D_{2} \otimes D_{1}\right),\left\{i, i+n_{2}+1\right\}\right) .
$$

We obtain $i=j$ and $n_{1}=n_{2}$. We denote $K_{1}=\Gamma_{t, j, \tau}^{1}\left(D_{1} \otimes D_{2}\right)$ and $K_{2}=$ $\Gamma_{p, i, \varrho}^{1}\left(D_{2} \otimes D_{1}\right)$, we consider the subgraph of $K_{1}$ (respectively $K_{2}$ ) formed by vertices in $\{1, \ldots, j\} \cup\left\{j+n_{1}+1, \ldots, j+2 n_{1}+1\right\}$ and we contract $\left\{j, j+n_{1}+1\right\}$. We then obtain $D_{1}=D_{2}$.

We now assume $D=D_{1}=D_{2}$.

5. It is sufficient to consider the proof of 1 .

6. It is true by definition.

7. It is trivial because $j$ is positive.

By direct calculation, we prove the following corollary.

Corollary 45 Let $D_{1}$ and $D_{2}$ be two dissection diagrams of positive degree $n_{1}$ and $n_{2}$. We define $\sigma_{D}$ as the number of different couples $(G, a)$ where $G$ is a dissection diagram of degree $n_{1}+n_{2}+1$ and a a chord $a=\{u, v\}$ with $0 \leq u<v \leq n_{1}+n_{2}+1$ obtained by applying operations 3 and 4 on $D_{1}$ and $D_{2}$. We have:

$$
\begin{aligned}
\sigma & \left(D_{1}, D_{2}\right) \\
& = \begin{cases}\left(v_{D_{2}}(0)+1\right) \sum_{i=0}^{n_{1}}\left(v_{D_{1}}(i)+1\right)+\left(v_{D_{1}}(0)+1\right) \sum_{j=0}^{n_{2}}\left(v_{D_{2}}(j)+1\right) & \text { if } D_{1} \neq D_{2}, \\
\left(v_{D_{2}}(0)+1\right) \sum_{i=0}^{n_{1}}\left(v_{D_{1}}(i)+1\right) & \text { if } D_{1}=D_{2},\end{cases} \\
& = \begin{cases}\left(v_{D_{2}}(0)+1\right)\left(3 n_{1}+1\right)+\left(v_{D_{1}}(0)+1\right)\left(3 n_{2}+1\right) & \text { if } D_{1} \neq D_{2}, \\
\left(v_{D_{1}}(0)+1\right)\left(3 n_{1}+1\right) & \text { if } D_{1}=D_{2} .\end{cases}
\end{aligned}
$$

Proposition 46 Let $D_{1}$ and $D_{2}$ be two dissection diagrams of positive degree $n_{1}$ and $n_{2}$.

$$
L\left(D_{1} D_{2}\right)=\sum_{\substack{t \in \llbracket 0, v_{D_{1}}(0) \rrbracket \\ j \in \llbracket 1, n_{2} \rrbracket \\ \tau \in \llbracket 0, v_{D_{2}}(j) \rrbracket}} x^{k_{\left\{a_{j, n_{1}}\right\}}\left(\Gamma_{t, j, \tau}^{1}\left(D_{1} \otimes D_{2}\right)\right)} \Gamma_{t, j, \tau}^{1}\left(D_{1} \otimes D_{2}\right)
$$




$$
\begin{aligned}
+ & \sum_{\substack{\tau \in \llbracket 0, v_{D_{2}}(0) \rrbracket \\
i \in \llbracket 1, n_{1} \rrbracket \\
t \in \llbracket 0, v_{D_{1}}(i) \rrbracket}} x^{\left.\left.k_{\left\{a_{i, n_{2}}\right\}}\right\} \Gamma_{\tau, i, t}^{1}\left(D_{2} \otimes D_{1}\right)\right)} \Gamma_{\tau, i, t}^{1}\left(D_{2} \otimes D_{1}\right) \\
+ & \sum_{\substack{\lambda \in\{0,1\} \\
t \in \llbracket 0, v_{D_{1}}(0) \rrbracket \\
\tau \in \llbracket 0, v_{D_{2}}(0) \rrbracket}} \Gamma_{t, \tau}^{\lambda}\left(D_{1} \otimes D_{2}\right)
\end{aligned}
$$

with

$$
a_{j, n_{1}}=\left\{j, j+n_{1}+1\right\} \text { and } a_{i, n_{2}}=\left\{i, i+n_{2}+1\right\} .
$$

In other words,

$$
\begin{aligned}
L\left(D_{1} D_{2}\right)= & \sum_{\substack{t \in \llbracket 0, v_{D_{1}}(0) \rrbracket \\
j \in \llbracket 1, n_{2} \rrbracket \\
\tau \in \llbracket 0, l_{2}(j)-1 \rrbracket}} \Gamma_{t, j, \tau}^{1}\left(D_{1} \otimes D_{2}\right)+x \sum_{\substack{t \in \llbracket 0, v_{D_{1}}(0) \rrbracket \\
j \in \llbracket 1, n_{2} \rrbracket \\
\tau \in \llbracket l_{2}(j), v_{D_{2}}(j) \rrbracket}} \Gamma_{t, j, \tau}^{1}\left(D_{1} \otimes D_{2}\right) \\
+ & \sum_{\substack{\tau \in \rrbracket 0, v_{D_{2}}(0) \rrbracket \\
i \in \llbracket 1, n_{1} \rrbracket \\
t \in \llbracket 0, l_{1}(i)-1 \rrbracket}} \Gamma_{\substack{\tau, i, t \\
\tau \in \llbracket 0, v_{D_{2}}(0) \rrbracket \\
i \in \llbracket 1, n_{1} \rrbracket \\
t \in \llbracket l_{1}(i), v_{D_{1}}(i) \rrbracket}}^{1}\left(D_{2} \otimes D_{1}\right)+x \Gamma_{\tau, i, t}^{1}\left(D_{2} \otimes D_{1}\right) \\
+ & \sum_{\substack{\lambda \in\{0,1\} \\
t \in \llbracket 0, v_{D_{1}}(0) \rrbracket \\
\tau \in \llbracket 0, v_{D_{2}}(0) \rrbracket}} \Gamma_{t, \tau}^{\lambda}\left(D_{1} \otimes D_{2}\right)
\end{aligned}
$$

where for any couples $(i, j) \in \llbracket 1, n_{1} \rrbracket \times \llbracket 1, n_{2} \rrbracket,\left(l_{1}(i), l_{2}(j)\right)$ is the unique couple of integers in $\llbracket 0, v_{D_{1}}(i) \rrbracket \times \llbracket 0, v_{D_{2}}(j) \rrbracket$ such that $a_{D_{1}, l_{1}(i)}^{i}$ in $\mathscr{C}\left(D_{1}\right)$ is labeled by $i$ and $a_{D_{2}, l_{2}(j)}^{j}$ in $\mathscr{C}\left(D_{2}\right)$ is labeled by $j$.

Proof We recall that, if $I d$ is the identity morphism and $l$ is the projection on $\mathcal{D}^{+}$, we have:

$$
L\left(D_{1} D_{2}\right)= \begin{cases}\sum_{\substack{G \in \mathcal{D} \\ \text { diagram }}}\left(Z_{D_{1} D_{2}} \otimes Z\right. \\ 2 \sum_{\substack{G \in \mathcal{D} \\ G \text { diagram }}}\left(Z_{D_{1} D_{2}} \otimes Z\right.\end{cases}
$$

Let $(G, a) \in(\mathcal{D})_{n_{1}+n_{2}+1} \times \mathscr{C}(G)$ be a couple diagram-chord such that $q_{\{a\}}(G)=$ $D_{1} D_{2}$. We write $a$ as $a=\{i, j\}$ with $0 \leq i<j \leq n_{1}+n_{2}+1$. There exists 
$v \in \llbracket 1, v_{G}(i) \rrbracket$ such that $a_{v}^{i}=\{i, j\} . q_{a}(G)=D_{1} D_{2}$, so $j=i+n+1$ with $n \in\left\{n_{1}, n_{2}\right\}$.

Case $1 i \neq 0$ We consider the subgraph $S$ of $G$ with vertices

$$
\{0, \ldots, i\} \cup\left\{j, \ldots, n_{1}+n_{2}+1\right\} .
$$

We have:

$$
\begin{aligned}
& (G, a) \\
& =\left\{\begin{array}{l}
\left(\Gamma_{t, i, \tau}^{1}\left(D_{1} \otimes D_{2}\right),\left\{i, i+n_{1}+1\right\}\right) \text { with } t=v_{D_{1}}(0)-v_{G}(i)+v \text { and } \tau=v-1 \text { if } q_{\{a\}}(S)=D_{2}, \\
\left(\Gamma_{\tau, i, t,}^{1}\left(D_{2} \otimes D_{1}\right),\left\{i, i+n_{2}+1\right\}\right) \text { with } t=v-1 \text { and } \tau=v_{D_{2}}(0)-v_{G}(i)+v \text { if } q_{\{a\}}(S)=D_{1} .
\end{array}\right.
\end{aligned}
$$

Case $2 \boldsymbol{i}=\mathbf{0}$ We use the subgraph $S$ of $G$ with vertices $\{0, \ldots, j\}$. We have:

$$
\begin{aligned}
& (G, a) \\
& \quad=\left\{\begin{array}{l}
\left(\Gamma_{t, \tau}^{1}\left(D_{1} \otimes, D_{2}\right),\left\{0, n_{1}+1\right\}\right) \text { with } t=v_{D_{1}}(0)-v_{G}(0)+v \text { and } \tau=v-1 \text { if } q_{\{a\}}(S)=D_{1}, \\
\left(\Gamma_{t, \tau}^{0}\left(D_{1} \otimes D_{2}\right),\left\{0, n_{2}+1\right\}\right) \text { with } t=v-1 \text { and } \tau=v_{D_{2}}(0)-v_{G}(0)+v \text { if } q_{\{a\}}(S)=D_{2}
\end{array}\right.
\end{aligned}
$$

Corollary 47 We assume that $x \in \mathbb{N}$. Let $D_{1}$ and $D_{2}$ be two dissection diagrams of positive degree $n_{1}$ and $n_{2}$. We call $\sigma_{L\left(D_{1} D_{2}\right)}$ the number of terms in $L\left(D_{1} D_{2}\right)$ counted with multiplicity.

$$
\begin{aligned}
\sigma_{L\left(D_{1} D_{2}\right)}= & \left(v_{D_{2}}(0)+1\right)\left[x\left(3 n_{1}+1\right)+(1-x) \sum_{i=1}^{n_{1}} l_{1}(i)+(1-x)\left(v_{D_{1}}(0)+1\right)\right] \\
& +\left(v_{D_{1}}(0)+1\right)\left[x\left(3 n_{2}+1\right)+(1-x) \sum_{j=1}^{n_{2}} l_{2}(j)+(1-x)\left(v_{D_{2}}(0)+1\right)\right]
\end{aligned}
$$

where, for any $(i, j) \in \llbracket 1, n_{1} \rrbracket \times \llbracket 1, n_{2} \rrbracket,\left(l_{1}(i), l_{2}(j)\right)$ is the unique element in $\llbracket 0, v_{D_{1}}(i) \rrbracket \times \llbracket 0, v_{D_{2}}(j) \rrbracket$ such that the chord $a_{D_{1}, l_{1}(i)}^{i}$ of $D_{1}$ is labeled by $i$ and the chord $a_{D_{2}, l_{2}(j)}^{j}$ of $D_{2}$ is labeled by $j$.

Proposition 48 Let $x$ be a scalar. Let $D, D_{1}, D_{2}, G_{1}$ and $G_{2}$ be five non-empty dissection diagrams. Then,

1. $L(D) \neq L\left(G_{1} G_{2}\right)$,

2. $L\left(D_{1} D_{2}\right)=L\left(G_{1} G_{2}\right) \Longleftrightarrow\left(\left(D_{1}, D_{2}\right)=\left(G_{1}, G_{2}\right)\right.$ or $\left.\left(D_{1}, D_{2}\right)=\left(G_{2}, G_{1}\right)\right)$.

Proof Let $x$ be a scalar in $\mathbb{K}$. Let $D, D_{1}, D_{2}, G_{1}$ and $G_{2}$ five dissection diagrams. We consider their root valency. As in the proof of Proposition 41, if $v_{D}(0) \neq v_{G_{1}}(0)+v_{G_{2}}(0)$ and if $v_{D_{1}}(0)+v_{D_{2}}(0) \neq v_{G_{1}}(0)+v_{G_{2}}(0)$, then $L(D) \neq L\left(G_{1} G_{2}\right)$ and $L\left(D_{1} D_{2}\right) \neq L\left(G_{1} G_{2}\right)$. We consider the case where $v_{D}(0)=v_{G_{1}}(0)+v_{G_{2}}(0)$ and $v_{D_{1}}(0)+v_{D_{2}}(0)=v_{G_{1}}(0)+v_{G_{2}}(0)$. We write 
the dissection diagrams as $D=(A), D_{1}=\left(\begin{array}{ccc}A \\ A_{1}\end{array}\right.$
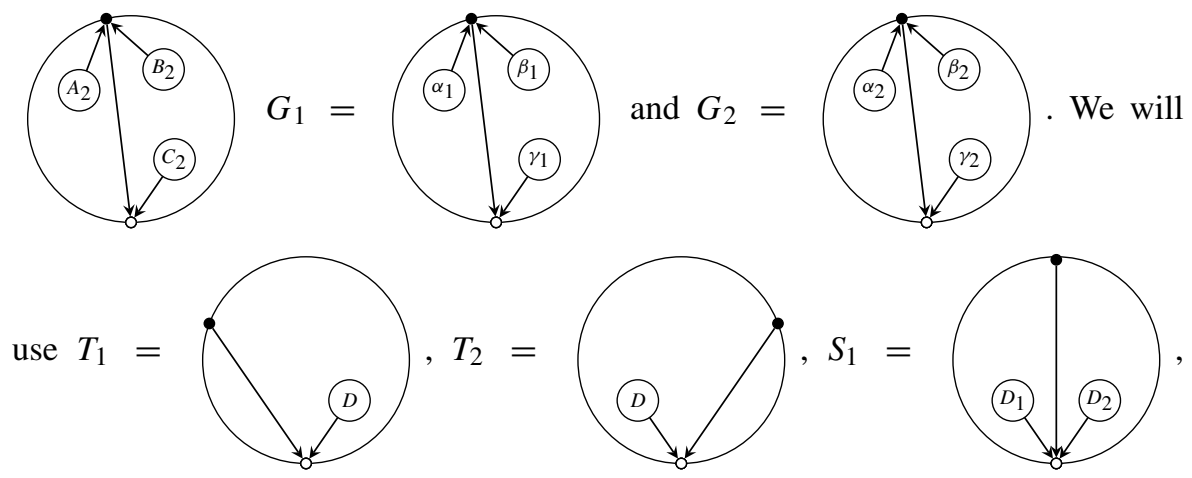

$S_{2}=\left(P_{1} P_{1}=\left(P_{1} P_{2}=\left(G_{1}\right), H_{1}=\right.\right.$
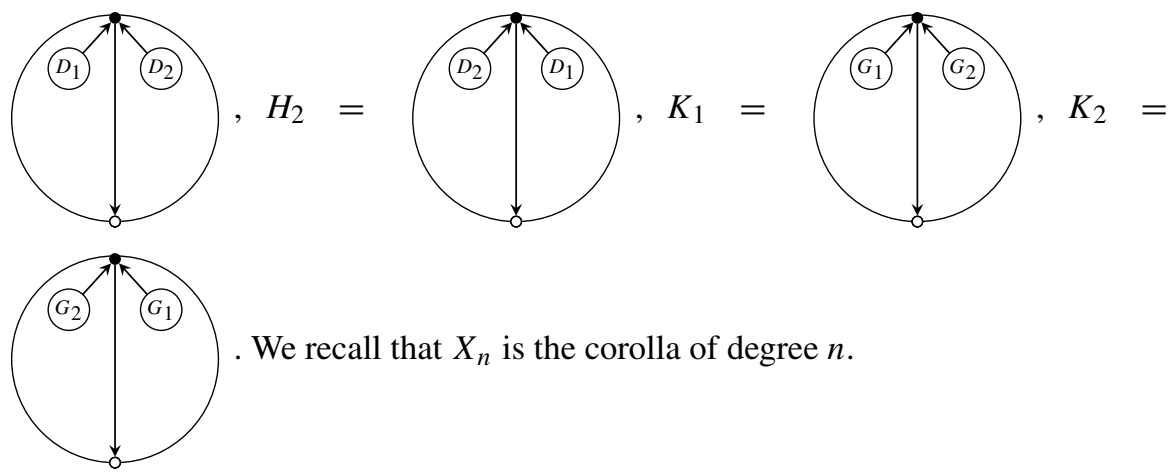

1. We introduce $J_{1}=$ $L(D)$ and $L\left(G_{1} G_{2}\right)$ equal, so $v_{D}(0) \geq 2$. There are two cases:

(a) If $v_{G_{1}}(0)$ and $v_{G_{2}}(0)$ are both different from 1 , then there exists an integer $i \in\{1,2\}$ such that $J_{1}=K_{i}$. It is impossible. 
(b) We now assume that there exists at least one integer $i \in\{1,2\}$ such that $v_{G_{i}}(0)=1$. Without loss of generality, we can consider $i=1$, so $\gamma_{1}$ is empty. We use know the fact that $\left\{T_{1}, T_{2}\right\}$ and $\left\{P_{1}, P_{2}\right\}$ be equal. We have two cases.

i. In the first one, $\left(T_{1}, T_{2}\right)$ equals $\left(P_{1}, P_{2}\right)$ so $\alpha_{1}$ and $\beta_{1}$ are empty, $G_{1}=X_{1}$, $A=\alpha_{2}, B=\beta_{2}$ and we have more

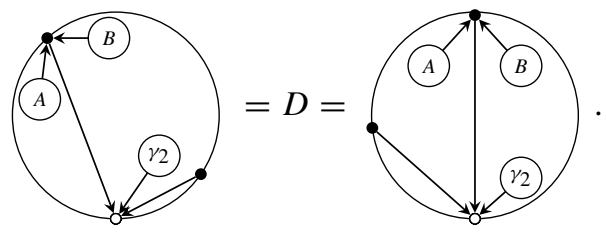

Thereafter, $A$ and $B$ are empty and we have the equality

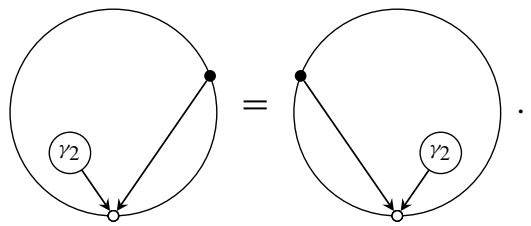

So, there exists a nonnegative integer $n$ such that $\gamma_{2}=X_{n}$ and then $D=X_{n+2}, G_{1}=X_{1}$ and $X_{n+1}$. It is impossible.

ii. In the second one, $\left(T_{1}, T_{2}\right)$ equals $\left(P_{2}, P_{1}\right)$ so $\alpha_{2}$ and $\beta_{2}$ are empty, $A=\alpha_{1}$, $B=\beta_{1}$ and we have two equalities

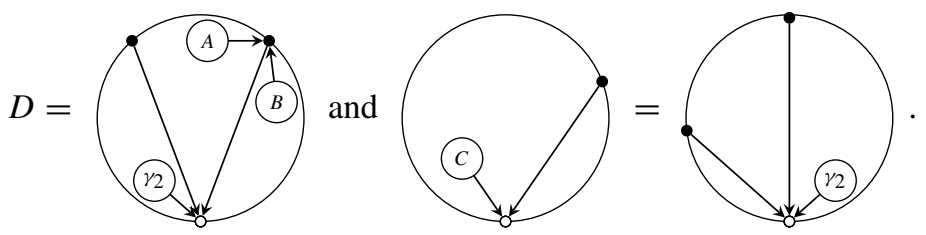

So, we obtain

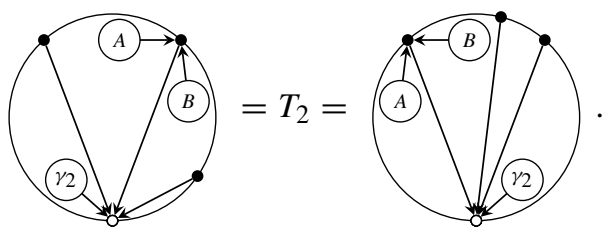

If $\gamma_{2}$ is empty, then $A$ and $B$ are empty too and we obtain $D=X_{2}$ and $G_{1}=G_{2}=X_{1}$. It is impossible. The diagram $\gamma_{2}$ is not empty. As for any integers $i$ and $j$ in $\{1,2\}$, the dissection diagrams $J_{i}$ and $K_{j}$ are differents, there exist two non-empty dissection diagrams $W_{1}$ and $W_{2}$ such 


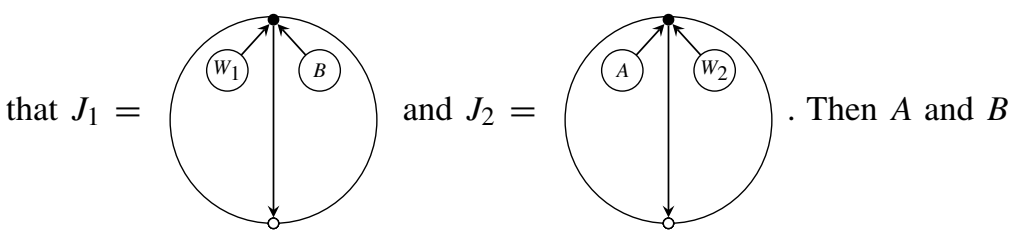

are empty and we obtain that $D, G_{1}$ and $G_{2}$ are corollas. It is impossible.

Thus, $L(D)$ and $L\left(G_{1} G_{2}\right)$ are different.

2. We assume $L\left(D_{1} D_{2}\right)$ and $L\left(G_{1} G_{2}\right)$ equal. We have to consider three cases.

(a) In the first case, $v_{D_{1}}(0), v_{D_{2}}(0), v_{G_{1}}(0)$ and $v_{G_{2}}(0)$ are all equal to 1 . As the two sets $\left\{S_{1}, S_{2}\right\}$ and $\left\{P_{1}, P_{2}\right\}$ are equal, the result is trivial.

(b) In the second case, $v_{D_{1}}(0), v_{D_{2}}(0), v_{G_{1}}(0)$ and $v_{G_{2}}(0)$ are all positive integers different from 1 . As the two sets $\left\{H_{1}, H_{2}\right\}$ and $\left\{K_{1}, K_{2}\right\}$ are equal, the result is trivial.

(c) In the third case, $v_{D_{1}}(0)=1$ and $v_{D_{2}}(0) \neq 1$. The sets $\left\{S_{1}, S_{2}\right\}$ and $\left\{P_{1}, P_{2}\right\}$ are equal, so we assume without loss of generality that $S_{1}=P_{1}$ and $S_{2}=P_{2}$. For any $i \in\{1,2\}$, we have $A_{i}=\alpha_{i}$ and $B_{i}=\beta_{i}$.

We start by proving that there exists a unique integer $i \in\{1,2\}$ such that $v_{G_{i}}(0)=1$. We need only prove existence. We assume $v_{G_{1}}(0)$ and $v_{G_{2}}(0)$ different from 1 . We consider $\Omega_{1}$ (respectively $\Omega_{2}$ ) the set of dissection diagrams of valency $v_{D_{1}}(0)+v_{D_{2}}(0)$ in the root built to obtain $L\left(D_{1} D_{2}\right)$ (respectively $\left.L\left(G_{1} G_{2}\right)\right)$. By writing $\gamma_{2}=\left(\right.$ with $\overline{\gamma_{2}}$ of valency 1 in the root, we have two different possibilities; either $\Omega_{1,1}=$

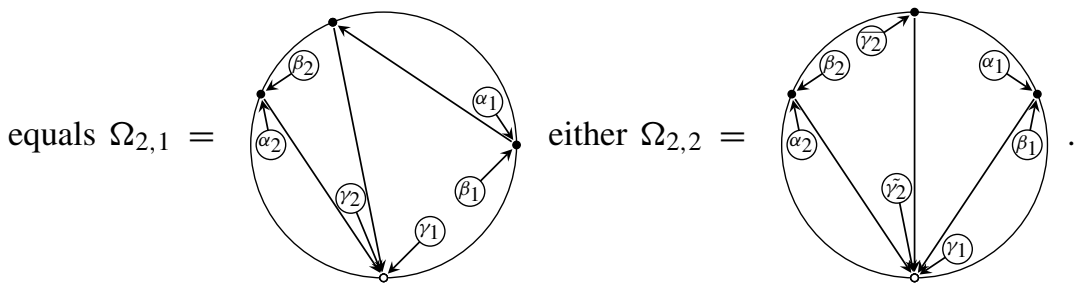

So, $B_{2}=\beta_{2}$ is empty and $A_{2}\left(=\alpha_{2}\right)$ and $D_{1}$ have the same degree. We consider $K_{1}$ and $K_{2}$. If there exists $i \in\{1,2\}$ such that $K_{1}=H_{i}$ or $K_{2}=H_{i}$ the one of the two couples $\left(G_{1}, G_{2}\right)$ and $\left(G_{2}, G_{1}\right)$ equals $\left(D_{i}, D_{-i+3}\right)$. It is 
not consistent. Thus, there exists a non-empty dissection diagram $W$ such that

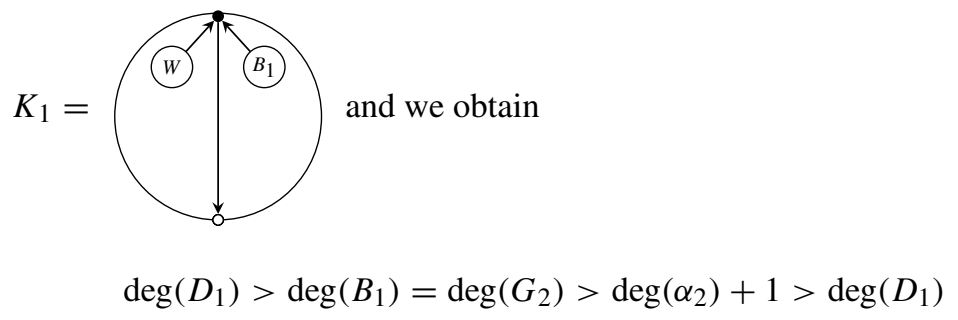

which is impossible.

We thus know that there exists a unique integer $i \in\{1,2\}$ such that $v_{G_{i}}(0)=1$. If $i=1$, then the result is trivial. If $i=2$, then we
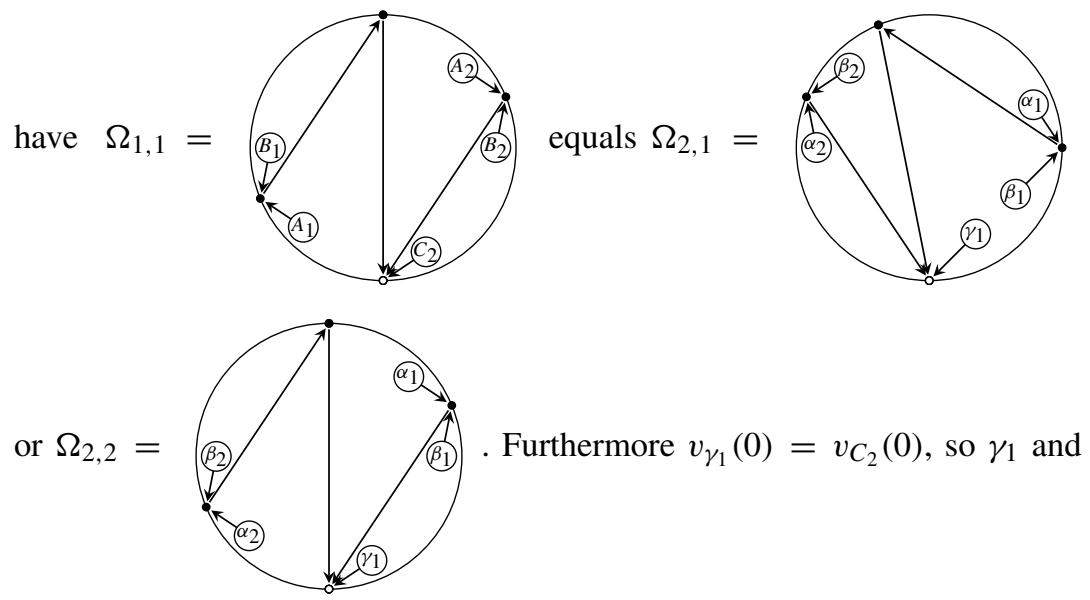

$C_{2}$ are equal. If $\Omega_{1,1}=\Omega_{2,2}$, then $A_{1}=A_{2}=\alpha_{1}=\alpha_{2}$ and $B_{1}=B_{2}=$ $\beta_{1}=\beta_{2}$, so $\left(D_{1}, D_{2}\right)=\left(G_{2}, G_{1}\right)$. If $\Omega_{1,1} \neq \Omega_{2,2}$, then $\Omega_{1,1}=\Omega_{2,1}$ and

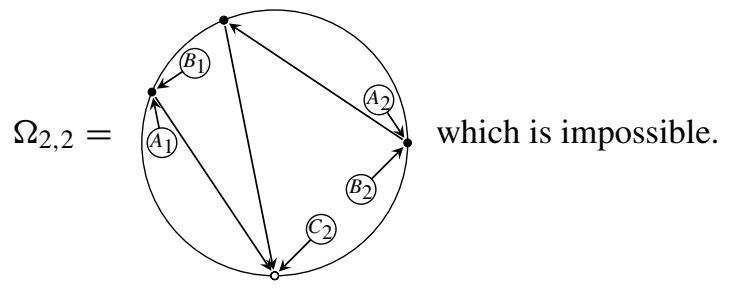

We have proved the statement

$$
\begin{aligned}
& L\left(D_{1} D_{2}\right)=L\left(G_{1} G_{2}\right) \\
& \quad \Longleftrightarrow\left(\left(D_{1}, D_{2}\right)=\left(G_{1}, G_{2}\right) \text { or }\left(D_{1}, D_{2}\right)=\left(G_{2}, G_{1}\right)\right) .
\end{aligned}
$$

Proposition 49 Let $n$ be a positive integer. There exist a basis $\mathcal{B}_{1}$ of sub-binary forests and a basis $\mathcal{B}_{2}$ of the dissection diagrams algebra such that the matrix in those bases of the restriction of $\varphi$ on homogeneous elements of degree $n$ is triangular by blocks. 
Proof Let $n$ be a positive integer and $t$ a sub-binary tree of degree $n$. We call $m(t)$ the number of vertices of $t$ with two children. For any disjoint union of dissection diagram $U=D_{1} \ldots D_{k}$, the integer $k$ is called length of $U$ and is denoted by $\mu(U)$. Let $D$ be a dissection diagram of degree $n$. We call $m(D)=\max \left\{\mu\left(q_{C}(D)\right), C \in \mathscr{C}(D)\right\}$. By definition of $L$, if $m(D)<m(t)$, then $Z_{D}(\varphi(t))=0$.

Corollary 50 Let $n$ be a positive integer. We recall that $e_{n} \in \mathcal{T}_{\mathrm{R}}$ is the ladder of degree $n, Y_{n} \in \mathcal{D}$ is the path tree of degree $n$ and $X_{n} \in \mathcal{D}$ is the corolla of degree $n$. Let $t$ be a sub-binary tree. We have $Z_{Y_{n}}(\varphi(t))=\left\{\begin{array}{l}n ! \text { if } t=e_{n}, \\ 0 \text { else }\end{array}\right.$ and $Z_{X_{n}}(\varphi(t)) \neq 0$. Actually $Z_{X_{n}}(\varphi(t))=2^{\operatorname{int}(t)}$ where $\operatorname{int}(t)$ is the number of internal vertices i.e. the number of vertices with at least one child.

Remark By considering $\varphi$ as the Hopf algebra morphism from SBT to $\mathcal{H}_{\mathrm{D}}^{\circledast}$, we can reformulate Conjecture 35 as follows: $\varphi$ is injective. Results 41,48 and 49 suggest that the conjecture is true. Direct calculations prove that the morphism $\varphi$, when restricted to degrees 1,23 or 4 , is injective.

Acknowledgements I would like to thank Université du Littoral Côte d'Opale (Grant No. 50\%) and the région Hauts-de-France (Grant No. 50\%) for their financial support. I would like to thank my supervisor for his support, Pierre-Louis GISCARD for his remarks and the two referees for their useful comments and suggestions.

\section{References}

1. Burde, D.: Left-symmetric algebras, or pre-Lie algebras in geometry and physics. Cent. Eur. J. Math. 4, 323-357 (2006)

2. Burgunder, E., Delcroix-Oger, B., Manchon, D.: An operad is never free as a pre-Lie algebra (2017, to be published)

3. Chapoton, F.: On some anticyclic operads. Algebr. Geom. Topol. 5, 53-69 (2005)

4. Chapoton, F.: Free pre-Lie algebras are free as lie algebras. Canad. Math. Bull. 53, 425-437 (2010)

5. Chapoton, F., Livernet, M.: Pre-Lie algebras and the rooted trees operad. Int. Math. Res. Notices 8, 395-408 (2001)

6. Connes, A., Kreimer, D.: Hopf algebras, renormalization and noncommutative geometry. In: DewittMorette, C., Zuber, J.-B. (eds.) Quantum Field Theory: Perspective and Prospective, vol. 530, pp. 59-109. Springer, Dordrecht (1999)

7. Doubilet, P., Rota, G.-C., Stanley, R.: On the foundations of combinatorial theory. VI. The idea of generating function. In: Proceedings of the Sixth Berkeley Symposium on Mathematical Statistics and Probability (Univ. California, Berkeley, Calif., 1970/1971), Vol. II: Probability Theory, pp. 267-318. University of California Press, Berkeley, California (1972)

8. Dupont, C.: The combinatorial hopf algebra of motivic dissection polylogarithms. Adv. Math. 264, 646-699 (2014)

9. Dupont, C.: Périodes des arrangements d'hyperplans et coproduit motivique. Ph.D. thesis, Université Pierre et Marie Curie (2014)

10. Foissy, L.: Algèbres de Hopf Combinatoires. http://loic.foissy.free.fr/pageperso/Hopf.pdf

11. Foissy, L.: Finite-dimensional comodules over the Hopf algebra of rooted trees. J. Algebra 255, 89-120 (2002)

12. Foissy, L.: The Hopf algebra of Fliess operators and its dual pre-Lie algebra. Commun. Algebra 43, 4528-4552 (2015)

13. Frabetti, A., Manchon, D.: Five interpretations of Faà di Bruno's formula. In: Faà di Bruno Hopf Algebras, Dyson-Schwinger Equations, and Lie-Butcher Series, vol. 21 of IRMA Lectures in Mathematics and Theoretical Physics, European Mathematical Society, Zürich, pp. 91-147 (2015) 
14. Gerstenhaber, M.: The cohomology structure of an associative ring. Ann. Math. 78, 267-288 (1963)

15. Grossman, R., Larson, R.G.: Hopf-algebraic structure of families of trees. J. Algebra 126, 184-210 (1989)

16. Grossman, R., Larson, R.G.: Hopf-algebraic structure of combinatorial objects and differential operators. Israel J. Math. 72, 109-117 (1990)

17. Grossman, R., Larson, R.G.: Symbolic computation of derivations using labelled trees. J. Symbolic Comput. 13, 511-523 (1992)

18. Hoffman, M.E.: Combinatorics of rooted trees and Hopf algebras. Trans. Amer. Math. Soc. 355, 37953811 (2003). (electronic)

19. Livernet, M.: A rigidity theorem for pre-Lie algebras. J. Pure Appl. Algebra 207, 1-18 (2006)

20. Loday, J.-L., Ronco, M.: Combinatorial Hopf algebras. In: Quanta of Maths, vol. 11 of Clay Mathematical Proceedings, pp. 347-383. American Mathematical Society, Providence, RI (2010)

21. Mammez, C.: Deux exemples d'algèbres de Hopf d'extraction-contraction: mots tassés et diagrammes de dissection. Ph.D. thesis, Université du Littoral Côte d'Opale - LMPA Joseph Liouville (2017)

22. Mansuy, A.: Preordered forests, packed words and contraction algebras. J. Algebra 411, 259-311 (2014)

23. Oudom, J.-M., Guin, D.: On the Lie enveloping algebra of a pre-Lie algebra. J. K-theory 2, 147-167 (2008)

24. Panaite, F.: Relating the Connes-Kreimer and Grossman-Larson Hopf algebras built on rooted trees. Lett. Math. Phys. 51, 211-219 (2000)

25. Sloane, N.J.A.: The On-line Encyclopedia of Integer Sequences. http://www.oeis.org (1964)

26. Vallette, B.: Homology of generalized partition posets. J. Pure Appl. Algebra 208, 699-725 (2007)

27. Vinberg, E.B.: The theory of homogeneous convex cones. Trudy Moskov. Mat. Obšč. 12, 303-358 (1963)

Publisher's Note Springer Nature remains neutral with regard to jurisdictional claims in published maps and institutional affiliations.

\section{Affiliations}

\section{Cécile Mammez ${ }^{1,2}$}

¿ Cécile Mammez

mammez@1mpa.univ-littoral.fr

1 EA 2597 - LMPA Laboratoire de Mathématiques Pures et Appliquées Joseph Liouville, Univ. Littoral Côte d'Opale, 62228 Calais, France

2 CNRS, FR 2956, Calais, France 